\title{
$X(3872)$ and Other Possible Heavy Molecular States
}

\author{
Xiang Liu ${ }^{1,2}$ * Zhi-Gang Luo ${ }^{1}$, Yan-Rui Liu ${ }^{3}$, and Shi-Lin Zhu1 ${ }^{1 \dagger}$ \\ ${ }^{1}$ Department of Physics, Peking University, Beijing 100871, China \\ ${ }^{2}$ Centro de Física Computacional, Departamento de Física, \\ Universidade de Coimbra, P-3004-516, Coimbra, Portugal \\ ${ }^{3}$ Institute of High Energy Physics, P.O. Box 918-4, Beijing 100049, China
}

(Dated: October 29, 2018)

\begin{abstract}
We perform a systematic study of the possible molecular states composed of a pair of heavy mesons such as $D \bar{D}, D^{*} \bar{D}, D^{*} \bar{D}^{*}$ in the framework of the meson exchange model. The exchanged mesons include the pseudoscalar, scalar and vector mesons. Through our investigation, we find that (1) the structure $X(3764)$ is not a molecular state; (2) There exists strong attraction in the range $r<1 \mathrm{fm}$ for the $D^{*} \bar{D}^{*}$ system with $J=0,1$. If future experiments confirm $Z^{+}(4051)$ as a loosely bound molecular state, its quantum number is probably $J^{P}=0^{+}$. Its partner state $\Phi^{* * 0}$ may be searched for in the $\pi^{0} \chi_{c 1}$ channel; (3) The vector meson exchange provides strong attraction in the $D^{*} \bar{D}$ channel together with the pion exchange. A bound state solution exists with a reasonable cutoff parameter $\Lambda \sim 1.4 \mathrm{GeV}$. X(3872) may be accommodated as a molecular state dynamically although drawing a very definite conclusion needs further investigation; (4) The $B^{*} \bar{B}$ molecular state exists.
\end{abstract}

PACS numbers: 12.39.Pn, 12.40.Yx, 13.75.Lb

\section{INTRODUCTION}

Since the observation of $Z^{+}$(4430) [1], the Belle Collaboration reported two new resonance-like structures $Z^{+}(4051)$ and $Z^{+}(4248)$ in the $\pi^{+} \chi_{c 1}$ mass distribution in the exclusive $\bar{B}^{0} \rightarrow K^{-} \pi^{+} \chi_{c 1}$ decay. Their masses and widths are $m_{Z^{+}(4051)}=\left(4051 \pm 14_{-41}^{+20}\right) \mathrm{MeV}, \Gamma_{Z^{+}(4051)}=\left(82_{-17-22}^{+21+47}\right) \mathrm{MeV}$ and $m_{Z^{+}(4248)}=\left(4248 \pm 14_{-29-35}^{+44+180}\right) \mathrm{MeV}, \Gamma_{Z^{+}(4248)}=$ $\left(177_{-39-61}^{+54+316}\right) \mathrm{MeV}$ [2]. These charged hidden charm signals are good candidates of either tetraquark states or heavy molecular states if they are confirmed by future experiments.

Recently the BES Collaboration reported an anomalous line-shape observed in the range of $3.650 \mathrm{GeV}$ to 3.872 $\mathrm{GeV}$ by analyzing the cross section of $e^{+} e^{-} \rightarrow$ hadrons. The anomalous line-shape is composed of two possible enhancement structures around $3.764 \mathrm{GeV}$ and $3.779 \mathrm{GeV}$ respectively [3]. The later one is consistent with the wellestablished $\psi(3770)$ while the mechanism of the first structure is not clear now, which is denoted as $X(3764)$ in this work.

In the past five years, a series of observations of the charmonium-like $X, Y, Z$ states, especially those states near the threshold of two charmed mesons have stimulated the interest in the possible existence of heavy molecular states greatly. The presence of the heavy quarks lowers the kinetic energy while the interaction between two light quarks could still provide strong enough attraction.

In fact, Voloshin and Okun studied the interaction between a pair of charmed mesons and proposed the possibilities of the molecular states involving charmed quarks more than thirty years ago [4]. De Rujula, Geogi and Glashow speculated $\psi(4040)$ as a $D^{*} \bar{D}^{*}$ molecular state [5]. Törnqvist studied the possible deuteron-like two-meson bound states such as $D \bar{D}^{*}$ and $D^{*} \bar{D}^{*}$ using the quark-pion interaction model [6]. Dubynskiy and Voloshin suggested the possibility of the existence of a new resonance at the $D^{*} \bar{D}^{*}$ threshold [7, 8]. Zhang, Chiang, Shen and Zou studied the possible $S$-wave bound states of two pseudoscalar mesons with the vector meson exchange in Ref. 9]. The experimental observation of $X(3872)$ and $Z^{+}(4430)$ motivated the extensive discussion of $X(3872)$ as a $D \bar{D}^{*}$ molecular state $10,11,12,13,14,15,16,17,18]$ and $Z^{+}(4430)$ as a $D_{1}^{\prime} D^{*}\left(D_{1} D^{*}\right)$ molecular state [19, 20, 21, 22].

It's interesting to note that the $Z^{+}(4051)$ enhancement announced by the Belle collaboration and the $X(3764)$ signal reported by the BES collaboration are near the thresholds of $D^{*} \bar{D}^{*}$ and $D \bar{D}$ respectively. One may wonder whether they could also be candidates of heavy molecular states. In this work we perform a systematic study of three types of possible heavy molecular states: the $D \bar{D} / B \bar{B}$ system (P-P), the $D^{*} \bar{D}^{*} / B^{*} \bar{B}^{*}$ system $(\mathrm{V}-\mathrm{V})$, and the $D^{*} \bar{D} / B^{*} \bar{B}$ system (P-V) using the formalism developed in Ref. [15, 21, 22].

*Electronic address: liuxiang@teor.fis.uc.pt

$\dagger$ Electronic address: zhusl@phy.pku.edu.cn 
This paper is organized as follows. After the introduction, we introduce some notations and present the flavor wave functions of the S-wave P-P, V-V and P-V systems constructed by two heavy flavor mesons. In Sec. III, we collect the effective Lagrangians in the derivation of the effective potential. Sections IV VI are for the P-P, V-V, P-V cases respectively. The last section is the discussion and conclusion.

\section{FLAVOR WAVE FUNCTIONS OF HEAVY MOLECULAR STATES}

We study the possible molecular states composed of two pseudoscalar (P-P) heavy mesons, two vector (V-V) heavy mesons, one pseudoscalar and one vector $(\mathrm{P}-\mathrm{V})$ heavy mesons. The masses of the $J^{P}=0^{-}, 1^{-}$heavy mesons are taken from PDG [23] and collected in Table I.

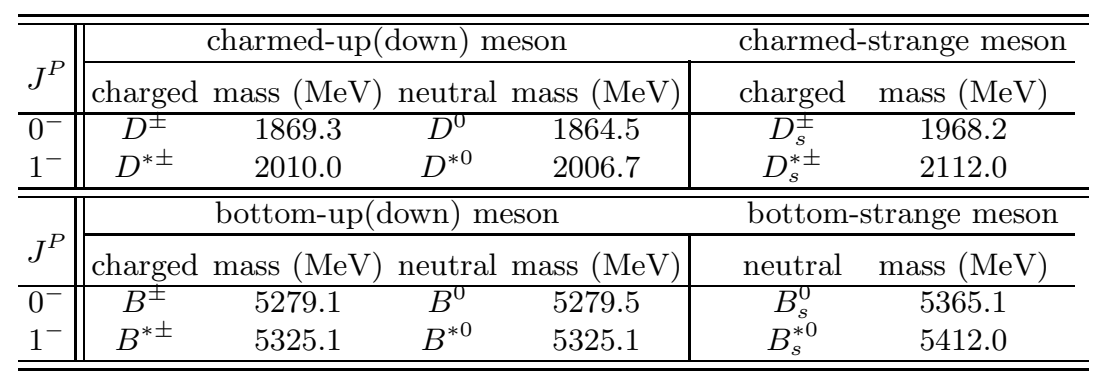

TABLE I: The masses of heavy mesons in the $\mathrm{H}$ doublet [23].

The P-P type is categorized as two systems, i.e. $\mathcal{D}-\overline{\mathcal{D}}, \overline{\mathcal{B}}-\mathcal{B}$. Here $\mathcal{D}, \overline{\mathcal{D}}, \mathcal{B}$ and $\overline{\mathcal{B}}$ denote $\left(D^{0}, D^{+}, D_{s}^{+}\right)$, $\left(\bar{D}^{0}, D^{-}, D_{s}^{-}\right),\left(B^{+}, B^{0}, B_{s}^{0}\right)$ and $\left(B^{-}, \bar{B}^{0}, \bar{B}_{s}^{0}\right)$ triplets respectively. In the following, we illustrate their flavor wave functions with the $\mathcal{D}-\overline{\mathcal{D}}$ type as an example. Since charmed mesons belong to the fundamental representation of flavor $S U(3)$, the $\mathcal{D}-\overline{\mathcal{D}}$ system form an octet and a singlet: $3 \otimes \overline{3}=8 \oplus 1$. The corresponding flavor wave functions are $\Phi_{s}^{+}=\bar{D}^{0} D_{s}^{+}, \Phi^{+}=\bar{D}^{0} D^{+}, \Phi_{s}^{0}=D^{-} D_{s}^{+}, \Phi^{0}=\frac{1}{\sqrt{2}}\left(\bar{D}^{0} D^{0}-D^{-} D^{+}\right), \bar{\Phi}_{s}^{0}=D_{s}^{-} D^{+}$, $\Phi_{8}=\frac{1}{\sqrt{6}}\left(\bar{D}^{0} D^{0}+D^{-} D^{+}-2 D_{s}^{-} D_{s}^{+}\right), \Phi^{-}=D^{-} D^{0}, \Phi_{s}^{-}=D_{s}^{-} D^{0}, \Phi_{1}=\frac{1}{\sqrt{3}}\left(\bar{D}^{0} D^{0}+D^{-} D^{+}+D_{s}^{-} D_{s}^{+}\right)$. Through ideal mixing, we have $\Phi_{8}^{0}=\frac{1}{\sqrt{2}}\left(\bar{D}^{0} D^{0}+D^{-} D^{+}\right)$and $\Phi_{s 1}^{0}=D_{s}^{-} D_{s}^{+}$, where $\Phi_{8}^{0}$ is an isoscalar while $\Phi^{0}$ is an isovector. The above states are shown in Fig. 1 and collected in Table $\llbracket$ together with the $\overline{\mathcal{B}}-\mathcal{B}$ type states. The flavor wave functions of the $D^{*} \bar{D}^{*}$ and $B^{*} \bar{B}^{*}$ systems are constructed similarly, which are denoted as $\Phi^{* *}$ and $\Omega^{* *}$ respectively.

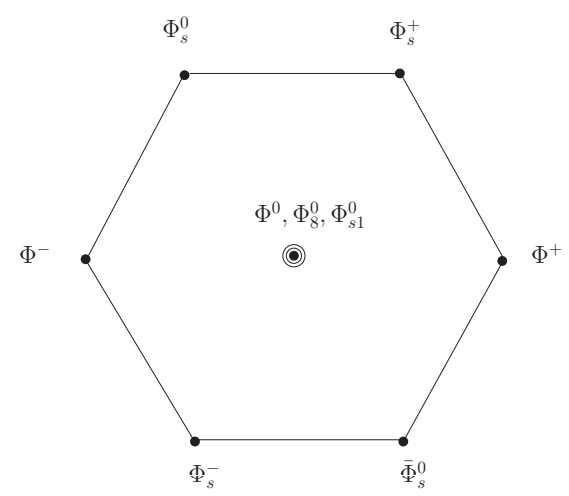

FIG. 1: The molecular multiplets composed of charmed and anit-charmed mesons.

We label the $\mathcal{D}-\overline{\mathcal{D}}^{*}$ and $\mathcal{B}-\overline{\mathcal{B}}^{*}$ systems as $\Phi^{*}$ and $\Omega^{*}$ respectively and list their flavor wave function in Table III. Here we need distinguish the $C$ parity for $\Phi^{* 0}, \Phi_{8}^{* 0}$ and $\Phi_{s 1}^{* 0}$ in the $\mathcal{D}-\overline{\mathcal{D}}^{*}$ system and $\Omega^{* 0}$, $\Omega_{8}^{* 0}$ and $\Omega_{s 1}^{* 0}$ in the $\mathcal{B}-\overline{\mathcal{B}}^{*}$ system. The parameter $c=\mp 1$ corresponds to $C= \pm 1$ respectively as pointed out in Ref. [15, 21]. In this work we label those states with the negative charge parity with a hat such as $\hat{\Phi}^{* 0}, \hat{\Phi}_{8}^{* 0}, \hat{\Phi}_{s 1}^{* 0}, \hat{\Omega}^{* 0}, \hat{\Omega}_{8}^{* 0}$ and $\hat{\Omega}_{s 1}^{* 0}$. The charge parity of $\mathrm{X}(3872)$ is positive. 


\begin{tabular}{c|c||c|c}
\hline \hline \multicolumn{1}{c}{$\mathcal{D}-\overline{\mathcal{D}}$} & \multicolumn{1}{c}{$\mathcal{B}$} \\
\hline State & wave function & State & wave function \\
\hline$\Phi_{s}^{+}$ & $\bar{D}^{0} D_{s}^{+}$ & $\Omega_{s}^{+}$ & $B^{+} \bar{B}_{s}^{0}$ \\
$\Phi^{+}$ & $\bar{D}^{0} D^{+}$ & $\Omega^{+}$ & $B^{+} \bar{B}^{0}$ \\
$\Phi_{s}^{0}$ & $D^{-} D_{s}^{+}$ & $\Omega_{s}^{0}$ & $B^{0} \bar{B}_{s}^{0}$ \\
$\Phi^{0}$ & $\frac{1}{\sqrt{2}}\left(\bar{D}^{0} D^{0}-D^{-} D^{+}\right)$ & $\Omega^{0}$ & $\bar{\Omega}_{s}^{0}$ \\
$\bar{\Phi}_{s}^{0}$ & $D_{s}^{-} D^{+}$ & $\Omega^{-}$ & $\frac{1}{\sqrt{2}}\left(B^{+} B^{-}-B^{0} \bar{B}^{0}\right)$ \\
$\Phi^{-}$ & $D^{-} D^{0}$ & $\Omega_{s}^{-}$ & $B_{s}^{0} \bar{B}^{0}$ \\
$\Phi_{s}^{-}$ & $D_{s}^{-} D^{0}$ & $\Omega_{8}^{0}$ & $B^{0} B^{-}$ \\
$\Phi_{8}^{0}$ & $\frac{1}{\sqrt{2}}\left(\bar{D}^{0} D^{0}+D^{-} D^{+}\right)$ & $\Omega_{s 1}^{0}$ & $B_{s}^{0} B^{-}$ \\
$\Phi_{s 1}^{0}$ & $D_{s}^{-} D_{s}^{+}$ & $\frac{1}{\sqrt{2}}\left(B^{+} B^{-}+B^{0} \bar{B}^{0}\right)$ \\
\hline \hline
\end{tabular}

TABLE II: The flavor wave functions of the $\mathcal{D}-\overline{\mathcal{D}}$ and $\mathcal{B}-\overline{\mathcal{B}}$ systems.

\begin{tabular}{|c|c|c|c|}
\hline \multicolumn{2}{|r|}{$\mathcal{D}-\overline{\mathcal{D}}^{*}$} & \multicolumn{2}{|r|}{$\mathcal{B}-\overline{\mathcal{B}}^{*}$} \\
\hline State & wave function & State & wave function \\
\hline$\Phi_{s}^{*+} / \hat{\Phi}_{s}^{*+}$ & $\frac{1}{\sqrt{2}}\left(\bar{D}^{* 0} D_{s}^{+}+c \bar{D}^{0} D_{s}^{*+}\right)$ & $\Omega_{s}^{*+} / \hat{\Omega}_{s}^{*+}$ & $\frac{1}{\sqrt{2}}\left(B^{*+} \bar{B}_{s}^{0}+c B^{+} \bar{B}_{s}^{* 0}\right)$ \\
\hline$\Phi^{*+} / \hat{\Phi}^{*+}$ & $\frac{1}{\sqrt{2}}\left(\bar{D}^{* 0} D^{+}+c \bar{D}^{0} D^{*+}\right)$ & $\Omega^{*+} / \hat{\Omega}^{*+}$ & $\frac{1}{\sqrt{2}}\left(B^{*+} \bar{B}^{0}+c B^{+} \bar{B}^{* 0}\right)$ \\
\hline$\Phi_{s}^{* 0} / \hat{\Phi}_{s}^{* 0}$ & $\frac{1}{\sqrt{2}}\left(D^{*-} D_{s}^{+}+c D^{-} D_{s}^{*+}\right)$ & $\Omega_{s}^{* 0} / \hat{\Omega}_{s}^{* 0}$ & $\frac{1}{\sqrt{2}}\left(B^{* 0} \bar{B}_{s}^{0}+c B^{0} \bar{B}_{s}^{* 0}\right)$ \\
\hline$\Phi^{* 0} / \hat{\Phi}^{* 0}$ & $\frac{1}{2}\left[\left(\bar{D}^{* 0} D^{0}-D^{*-} D^{+}\right)+c\left(\bar{D}^{0} D^{* 0}-D^{-} D^{*+}\right)\right]$ & $\Omega^{* 0} / \hat{\Omega}^{* 0}$ & $\frac{1}{2}\left[\left(B^{*+} B^{-}-B^{* 0} \bar{B}^{0}\right)+c\left(B^{+} B^{*-}-B^{0} \bar{B}^{* 0}\right)\right]$ \\
\hline $\bar{\Phi}_{s}^{* 0} / \hat{\bar{\Phi}}_{s}^{* 0}$ & $\frac{1}{\sqrt{2}}\left(D_{s}^{*-} D^{+}+c D_{s}^{-} D^{*+}\right)$ & $\bar{\Omega}_{s}^{* 0} / \hat{\bar{\Omega}}_{s}^{* 0}$ & $\frac{1}{\sqrt{2}}\left(B_{s}^{* 0} \bar{B}^{0}+c B_{s}^{0} \bar{B}^{* 0}\right)$ \\
\hline$\Phi^{*-} / \hat{\Phi}^{*-}$ & $\frac{1}{\sqrt{2}}\left(D^{*-} D^{0}+c D^{-} D^{* 0}\right)$ & $\Omega^{*-} / \hat{\Omega}^{*-}$ & $\frac{1}{\sqrt{2}}\left(B^{* 0} B^{-}+c B^{0} B^{*-}\right)$ \\
\hline$\Phi_{s}^{*-} / \hat{\Phi}_{s}^{*-}$ & $\frac{1}{\sqrt{2}}\left(D_{s}^{*-} D^{0}+c D_{s}^{-} D^{* 0}\right)$ & $\Omega_{s}^{*-} / \hat{\Omega}_{s}^{*-}$ & $\frac{1}{\sqrt{2}}\left(B_{s}^{* 0} B^{-}+c B_{s}^{0} B^{*-}\right)$ \\
\hline$\Phi_{8}^{* 0} / \hat{\Phi}_{8}^{* 0}$ & $\frac{1}{2}\left[\left(\bar{D}^{* 0} D^{0}+D^{*-} D^{+}\right)+c\left(\bar{D}^{0} D^{* 0}+D^{-} D^{*+}\right)\right]$ & $\Omega_{8}^{* 0} / \hat{\Omega}_{8}^{* 0}$ & $\frac{1}{2}\left[\left(B^{*+} B^{-}+B^{* 0} \bar{B}^{0}\right)+c\left(B^{+} B^{*-}+B^{0} \bar{B}^{* 0}\right)\right]$ \\
\hline$\Phi_{s 1}^{* 0} / \hat{\Phi}_{s 1}^{* 0}$ & $\frac{1}{\sqrt{2}}\left(D_{s}^{*-} D_{s}^{+}+c D_{s}^{-} D_{s}^{*+}\right)$ & $\Omega_{s 1}^{* 0} / \hat{\Omega}_{s 1}^{* 0}$ & $\frac{1}{\sqrt{2}}\left(B_{s}^{0} \bar{B}_{s}^{0}+c B_{s}^{0} \bar{B}_{s}^{* 0}\right)$ \\
\hline
\end{tabular}

TABLE III: The flavor wave functions of the $\mathcal{D}-\overline{\mathcal{D}}^{*}$ and $\mathcal{B}-\overline{\mathcal{B}}^{*}$ systems.

\section{THE EFFECTIVE LAGRANGIAN IN THE DERIVATION OF THE POTENTIAL}

In the derivation of the potential, we need the the effective Lagrangians, which are constructed based on the chiral symmetry and heavy quark symmetry: 25, 26, 27]

$$
\begin{aligned}
\mathcal{L}= & i g \operatorname{Tr}\left[H_{b} \gamma_{\mu} \gamma_{5} \mathcal{A}_{b a}^{\mu} \bar{H}_{a}\right]+i \beta \operatorname{Tr}\left[H_{b} v^{\mu}\left(\mathcal{V}_{\mu}-\rho_{\mu}\right)_{b a} \bar{H}_{a}\right] \\
& +i \lambda \operatorname{Tr}\left[H_{b} \sigma^{\mu \nu} F_{\mu \nu}(\rho) \bar{H}_{a}\right]+g_{\sigma} \operatorname{Tr}\left[H_{a} \sigma \bar{H}_{a}\right]
\end{aligned}
$$

where the field $H$ is defined in terms of the $\left(0^{-}, 1^{-}\right)$doublet

$$
H_{b}=\frac{1+\not k}{2}\left[P_{b}^{* \mu} \gamma_{\mu}+i P_{b} \gamma_{5}\right]
$$

$A_{a b}^{\mu}$ is the axial vector field with definition

$$
A_{a b}^{\mu}=\frac{1}{2}\left(\xi^{\dagger} \partial^{\mu} \xi-\xi \partial^{\mu} \xi^{\dagger}\right)_{a b}=\frac{i}{f_{\pi}} \partial^{\mu} \mathbb{P}_{a b}+\cdots
$$

with $\xi=\exp \left(i \mathbb{P} / f_{\pi}\right)$ and $f_{\pi}=132 \mathrm{MeV}$. The octet pseudoscalar and nonet vector meson matrices are

$$
\mathbb{P}=\left(\begin{array}{ccc}
\frac{\pi^{0}}{\sqrt{2}}+\frac{\eta}{\sqrt{6}} & \pi^{+} & K^{+} \\
\pi^{-} & -\frac{\pi^{0}}{\sqrt{2}}+\frac{\eta}{\sqrt{6}} & K^{0} \\
K^{-} & \bar{K}^{0} & -\frac{2 \eta}{\sqrt{6}}
\end{array}\right)
$$




$$
\mathbb{V}=\left(\begin{array}{ccc}
\frac{\rho^{0}}{\sqrt{2}}+\frac{\omega}{\sqrt{2}} & \rho^{+} & K^{*+} \\
\rho^{-} & -\frac{\rho^{0}}{\sqrt{2}}+\frac{\omega}{\sqrt{2}} & K^{* 0} \\
K^{*-} & K^{* 0} & \phi
\end{array}\right)
$$

The effective interaction Lagrangians at the tree level from Eq. (11) are

$$
\begin{aligned}
& \mathcal{L}_{\mathcal{D D V}}=-i g_{\mathcal{D D V}}\left(\mathcal{D}_{a}^{\dagger} \partial_{\mu} \mathcal{D}_{b}-\mathcal{D}_{b} \partial_{\mu} D_{a}^{\dagger}\right)\left(\mathbb{V}^{\mu}\right)_{a b}, \\
& \mathcal{L}_{\mathcal{D D} \sigma}=-2 m_{\mathcal{D}} g_{\sigma} \mathcal{D}_{a} \mathcal{D}_{a}^{\dagger} \sigma \\
& \mathcal{L}_{\mathcal{D}^{*} \mathcal{D}^{*} \mathbb{P}}=\frac{1}{2} g_{\mathcal{D}^{*} \mathcal{D}^{*} \mathbb{P}} \varepsilon_{\mu \nu \alpha \beta}\left(D_{a}^{* \mu} \partial^{\alpha} \mathcal{D}_{b}^{* \beta \dagger}-\mathcal{D}_{b}^{* \beta \dagger} \partial^{\alpha} \mathcal{D}_{a}^{* \mu}\right) \partial^{\nu} \mathbb{P}_{a b}, \\
& \mathcal{L}_{\mathcal{D}^{*} \mathcal{D}^{*} \mathbb{V}}=i g_{\mathcal{D}^{*} \mathcal{D} * \mathbb{V}}\left(\mathcal{D}_{a}^{* \nu \dagger} \partial^{\mu} \mathcal{D}_{\nu, b}^{*}-\mathcal{D}_{\nu b}^{*} \partial^{\mu} \mathcal{D}_{a}^{* \nu \dagger}\right)\left(\mathbb{V}_{\mu}\right)_{a b}+4 i f_{\mathcal{D}^{*} \mathcal{D} * \mathbb{V}} D_{\mu a}^{* \dagger} D_{\nu b}^{*}\left(\partial^{\mu} \mathbb{V}^{\nu}-\partial^{\nu} \mathbb{V}^{\mu}\right)_{a b}, \\
& \mathcal{L}_{\mathcal{D}^{*} \mathcal{D}^{*} \sigma}=2 m_{\mathcal{D}^{*}} g_{\sigma} \mathcal{D}_{a}^{* \alpha} \mathcal{D}_{\alpha a}^{* \dagger} \sigma, \\
& \mathcal{L}_{\mathcal{D}^{*} \mathcal{D P}}=-i g_{\mathcal{D}^{*} \mathcal{D P}}\left(\mathcal{D}_{a} \mathcal{D}_{\mu b}^{* \dagger}-\mathcal{D}_{\mu a}^{*} \mathcal{D}_{b}^{\dagger}\right) \partial^{\mu} \mathbb{P}_{a b}, \\
& \mathcal{L}_{\mathcal{D}^{*} \mathcal{D} \mathbb{V}}=-2 f_{\mathcal{D}^{*} \mathcal{D} \mathbb{V} \varepsilon_{\mu \nu \alpha \beta}}\left(\partial^{\mu} \mathbb{V}^{\nu}\right)_{a b}\left[\left(\mathcal{D}_{a}^{\dagger} \partial^{\alpha} \mathcal{D}_{b}^{* \beta}-\partial^{\alpha} \mathcal{D}_{a}^{\dagger} \mathcal{D}_{b}^{* \beta}\right)-\left(\mathcal{D}_{a}^{* \beta \dagger} \partial^{\alpha} \mathcal{D}_{b}-\partial^{\alpha} \mathcal{D}_{a}^{* \beta \dagger} \mathcal{D}_{b}\right)\right],
\end{aligned}
$$

where $\mathcal{D}^{(*)}=\left(\left(\bar{D}^{0}\right)^{(*)},\left(D^{-}\right)^{(*)},\left(D_{s}^{-}\right)^{(*)}\right)$. The relevant coupling constants are

$$
\begin{aligned}
g_{\mathcal{D}^{*} \mathcal{D}^{*} \mathbb{P}} & =\frac{g_{\mathcal{D}^{*} \mathcal{D} \mathbb{P}}}{\sqrt{m_{\mathcal{D} m_{\mathcal{D}^{*}}}}}=\frac{2 g}{f_{\pi}}, \quad g_{\mathcal{D} \mathcal{D} \mathbb{V}}=g_{\mathcal{D}^{*} \mathcal{D}^{*} \mathbb{V}}=\frac{\beta g_{V}}{\sqrt{2}}, \\
f_{\mathcal{D}^{*} \mathcal{D} \mathbb{V}} & =\frac{f_{\mathcal{D}^{*} \mathcal{D}^{*} \mathbb{V}}}{m_{\mathcal{D}^{*}}}=\frac{\lambda g_{V}}{\sqrt{2}}, \quad g_{V}=\frac{m_{\rho}}{f_{\pi}}, \quad g_{\sigma}=\frac{g_{\pi}}{2 \sqrt{6}},
\end{aligned}
$$

where $g_{V}, \beta$ and $\lambda$ are parameters in the effective chiral Lagrangian that describe the interaction of heavy mesons with light vector mesons [26]. Following Ref. [28], we take $g=0.59, \beta=0.9$ and $\lambda=0.56 \mathrm{GeV}^{-1} . g_{\pi}=3.73$ [27].

In this work, we adopt the same formalism developed in Refs. [15, 21, 22] to derive the effective potential. We compute the amplitudes of the elastic scattering of two heavy mesons using the above effective Lagrangians. In order to account for the structure effect of every interaction vertex, we introduce the monopole type form factor (FF) [6, 24]

$$
F(q)=\frac{\Lambda^{2}-m^{2}}{\Lambda^{2}-q^{2}}
$$

Here $\Lambda$ is the phenomenological parameter around $1 \mathrm{GeV}$, and $q$ denotes the four-momentum of the exchanged meson. The FF also plays the role of regularizing the potential by imposing a short-distance cutoff to cure the singularity of the effective potential. Then we impose the constraint that the initial states and final states should have the same angular momentum. After averaging the potentials obtained with the Breit approximation in the momentum space, we finally perform Fourier transformation to derive the potentials in the coordinate space. In the following sections, we illustrate the effective potentials in detail for the P-P, V-V and P-V systems.

\section{THE $\mathcal{D}-\overline{\mathcal{D}}$ CASE}

\section{A. The potential of the P-P system}

The quantum number of the $\mathrm{S}$-wave $\mathcal{D}-\overline{\mathcal{D}}$ system is $J^{P}=0^{+}$and the $\mathrm{C}$ parity of the neutral states is positive. In this work we consider the possible molecular states bound by the force from the light pseudoscalar meson, vector meson and scalar exchange. Parity and angular momentum conservation forbid the exchange of a pseudoscalar meson between the $\mathcal{D}-\overline{\mathcal{D}}$ pair. No suitable meson exchange is allowed for the $\Phi_{s}^{ \pm}$and $\Phi_{s}^{0}\left(\bar{\Phi}_{s}^{0}\right)$ cases. For the other states, the effective potentials in the momentum space from the vector meson and $\sigma$ meson exchange are

$$
\begin{aligned}
\mathcal{V}_{\mathrm{v}}(\mathbf{q})\left[I_{1}^{\mathbb{V} \mathcal{D}_{1} \mathcal{D}_{1}}, I_{2}^{\mathbb{V} \mathcal{D}_{2} \mathcal{D}_{2}}, m_{\mathcal{D}_{1}}, m_{\mathcal{D}_{2}}, m_{\mathbb{V}}\right] & =-g_{\mathcal{D} \mathcal{D} \mathbb{V}}^{2} I_{1}^{\mathbb{V} \mathcal{D}_{1} \mathcal{D}_{1}} I_{2}^{\mathbb{V} \mathcal{D}_{2} \mathcal{D}_{2}}\left(\frac{1}{\mathbf{q}^{2}+m_{\mathbb{V}}^{2}}+\frac{\mathbf{q}^{2}}{4 m_{\mathcal{D}_{1}} m_{\mathcal{D}_{2}} m_{\mathbb{V}}^{2}}\right), \\
\mathcal{V}_{\sigma}(\mathbf{q}) & =-g_{\sigma}^{2} \frac{1}{\mathbf{q}^{2}+m_{\sigma}^{2}},
\end{aligned}
$$


where $m_{\mathcal{D}}$ and $m_{\mathbb{V}}$ denote the masses of charmed meson and exchanged vector meson respectively. $I_{1}^{\mathbb{V} \mathcal{D}_{1} \mathcal{D}_{1}}$ and $I_{2}^{\mathbb{V} \mathcal{D}_{2} \mathcal{D}_{2}}$ arise from the coefficients related to the exchanged meson, which can be read from the nonet vector meson matrix in Eq. (5). After making the Fourier transformation, the three independent structures in Eqs. (8)-(9) read as

$$
\begin{gathered}
\mathbf{q}^{2} \longrightarrow X(\Lambda, m, r), \\
\frac{1}{\mathbf{q}^{2}+m^{2}} \longrightarrow Y(\Lambda, m, r), \\
\frac{\mathbf{q}^{2}}{\mathbf{q}^{2}+m^{2}} \longrightarrow Z(\Lambda, m, r),
\end{gathered}
$$

where

$$
\begin{aligned}
Y[\Lambda, m, r] & =\frac{1}{4 \pi r}\left(e^{-m r}-e^{-\Lambda r}\right)-\frac{\xi^{2}}{8 \pi \Lambda} e^{-\Lambda r} \\
Z[\Lambda, m, r] & =-\frac{1}{r^{2}} \frac{\partial}{\partial r}\left(r^{2} \frac{\partial}{\partial r}\right) Y[\Lambda, m, r] \\
X[\Lambda, m, r] & =\left[-\frac{1}{r^{2}} \frac{\partial}{\partial r}\left(r^{2} \frac{\partial}{\partial r}\right)+m^{2}\right] Z[\Lambda, m, r]
\end{aligned}
$$

with $\xi=\sqrt{\Lambda^{2}-m^{2}}$. We have adopted the monopole FF in Eq. (7) to regularize the potential in Eqs. (8)-(9). Now the effective potentials in the coordinate space read

$$
\begin{aligned}
\mathcal{V}_{\mathrm{V}}(r)\left[I_{1}^{\mathbb{V} \mathcal{D}_{1} \mathcal{D}_{1}}, I_{2}^{\mathbb{V} \mathcal{D}_{2} \mathcal{D}_{2}}, m_{\mathcal{D}_{1}}, m_{\mathcal{D}_{2}}, m_{\mathbb{V}}\right] & =-g_{\mathcal{D} \mathcal{D} \mathbb{V}}^{2} I_{1}^{\mathbb{V} \mathcal{D}_{1} \mathcal{D}_{1}} I_{2}^{\mathbb{V} \mathcal{D}_{2} \mathcal{D}_{2}}\left(Y\left[\Lambda, m_{\mathbb{V}}, r\right]+\frac{X\left[\Lambda, m_{\mathbb{V}}, r\right]}{4 m_{\mathcal{D}_{1}} m_{\mathcal{D}_{2}} m_{\mathbb{V}}^{2}}\right), \\
\mathcal{V}_{\sigma}(r) & =-g_{\sigma}^{2} Y\left[\Lambda, m_{\sigma}, r\right] .
\end{aligned}
$$

The exchange potential for $\Phi^{ \pm}$in coordinate space is

$$
\begin{aligned}
\mathcal{V}(r)_{\text {Total }}^{\Phi^{ \pm}} & =\mathcal{V}_{\mathbb{V}}(r)\left[\frac{1}{\sqrt{2}},-\frac{1}{\sqrt{2}}, m_{D^{0}}, m_{D^{+}}, m_{\rho^{0}}\right]+\mathcal{V}_{\mathbb{V}}(r)\left[\frac{1}{\sqrt{2}}, \frac{1}{\sqrt{2}}, m_{D^{0}}, m_{D^{+}}, m_{\omega}\right]+\mathcal{V}_{\sigma}(r) \\
& \approx-g_{\mathcal{D} \mathcal{D V}}^{2}\left[\frac{Y\left[\Lambda, m_{\omega}, r\right]}{2}-\frac{Y\left[\Lambda, m_{\rho}, r\right]}{2}-\frac{X\left[\Lambda, m_{\rho}, r\right]}{8 m_{D}^{2} m_{\rho}^{2}}+\frac{X\left[\Lambda, m_{\omega}, r\right]}{8 m_{D}^{2} m_{\omega}^{2}}\right]-g_{\sigma}^{2} Y\left[\Lambda, m_{\sigma}, r\right]
\end{aligned}
$$

The symbol " $\approx$ " means the $S U(2)$ symmetry is assumed. For the $\Phi^{0}$ and $\Phi_{8}^{0}$ states, the exchange potentials are

$$
\begin{aligned}
\mathcal{V}(r)_{\text {Total }}^{\Phi^{0}\left(\Phi_{8}^{0}\right)=} & \frac{1}{2}\left\{\mathcal{V}_{\mathbb{V}}(r)\left[\frac{1}{\sqrt{2}}, \frac{1}{\sqrt{2}}, m_{D^{0}}, m_{D^{0}}, m_{\rho^{0}}\right] \mp 2 \mathcal{V}_{\mathbb{V}}(r)\left[1,1, m_{D^{0}}, m_{D^{+}}, m_{\rho^{ \pm}}\right]\right. \\
& +\mathcal{V}_{\mathbb{V}}(r)\left[-\frac{1}{\sqrt{2}},-\frac{1}{\sqrt{2}}, m_{D^{+}}, m_{D^{-}}, m_{\rho^{0}}\right]+\mathcal{V}_{\mathbb{V}}(r)\left[\frac{1}{\sqrt{2}}, \frac{1}{\sqrt{2}}, m_{D^{0}}, m_{D^{0}}, m_{\omega}\right] \\
& \left.+\mathcal{V}_{\mathbb{V}}(r)\left[\frac{1}{\sqrt{2}}, \frac{1}{\sqrt{2}}, m_{D^{+}}, m_{D^{-}}, m_{\omega}\right]+2 \mathcal{V}_{\sigma}(r)\right\} \\
\approx & -\frac{g_{\mathcal{D} \mathcal{D V}}^{2}}{2}(1 \mp 2)\left(Y\left[\Lambda, m_{\rho}, r\right]+\frac{X\left[\Lambda, m_{\rho}, r\right]}{4 m_{D}^{2} m_{\rho}^{2}}\right)-\frac{g_{\mathcal{D} \mathcal{V} \mathbb{V}}^{2}}{2}\left(Y\left[\Lambda, m_{\omega}, r\right]+\frac{X\left[\Lambda, m_{\omega}, r\right]}{4 m_{D}^{2} m_{\omega}^{2}}\right) \\
& -g_{\sigma}^{2} Y\left[\Lambda, m_{\sigma}, r\right] .
\end{aligned}
$$

Clearly the potential of $\Phi_{0}$ is the same as that of $\Phi^{ \pm}$due to the $\mathrm{SU}(2)$ symmetry. The effective potential of $\Phi_{s 1}^{0}$ is

$$
\begin{aligned}
\mathcal{V}(r)_{\text {Total }}^{\Phi_{s 1}^{0}} & =\mathcal{V}_{\mathbb{V}}(r)\left[1,1, m_{D_{s}}, m_{D_{s}}, m_{\phi}\right] \\
& =-g_{\mathcal{D D V}}^{2}\left(Y\left[\Lambda, m_{\phi}, r\right]+\frac{X\left[\Lambda, m_{\phi}, r\right]}{4 m_{D_{s}}^{2} m_{\phi}^{2}}\right),
\end{aligned}
$$

where only the $\phi$ meson exchange is allowed. 


\section{B. Numerical results for the P-P system}

The input parameters include the coupling constants in Eq. (6), the masses of heavy mesons listed in Table 1 , and the exchanged meson masses $m_{\pi}=139.5 \mathrm{MeV}, m_{\rho}=775.5 \mathrm{MeV}, m_{\sigma}=600 \mathrm{MeV}, m_{\eta}=547.5 \mathrm{MeV}, m_{\omega}=782.7$ $\mathrm{MeV}$ and $m_{\phi}=1019.5 \mathrm{MeV}$.

We first plot the variation of the effective potentials of the $\mathcal{D}-\overline{\mathcal{D}}$ system with the cutoff $\Lambda=1 \mathrm{GeV}$ in Fig. 2 , For the $\Phi^{ \pm}\left(\Phi^{0}\right)$ state, there exist the $\rho, \omega$ and $\sigma$ meson exchanges. The $\rho$ exchange force is repulsive while both the $\omega$ and $\sigma$ meson exchange forces are attractive. The $\rho$ and $\omega$ exchange forces cancel each other almost exactly. Thus the total potential is attractive. For the state $\Phi_{s 1}^{0}$, the $\phi$ meson exchange potential is attractive. The exchange potentials of $\rho, \omega$ and $\sigma$ mesons all are attractive for $\Phi_{8}^{0}$. Thus its total effective potential is attractive.

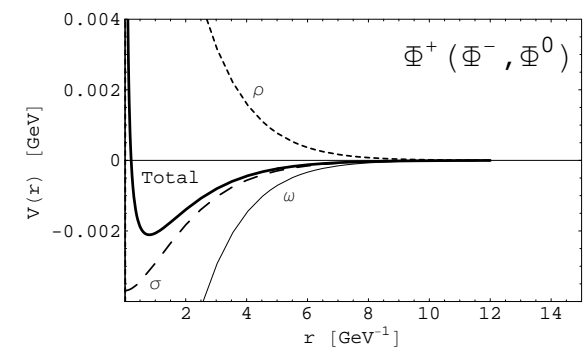

(a)

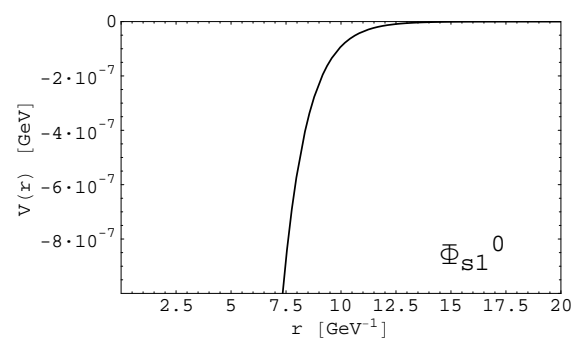

(b)

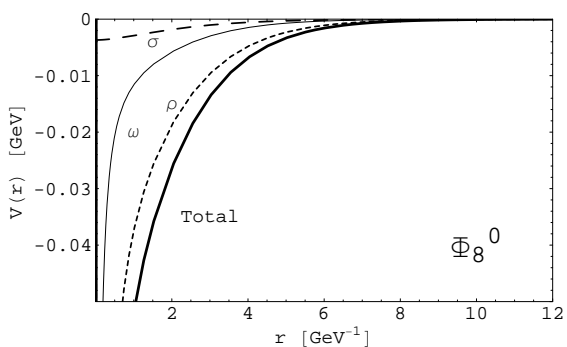

(c)

FIG. 2: Diagrams (a), (b) and (c) give respectively the variation of potentials of $\Phi^{ \pm}(\Phi)^{0}, \Phi_{s 1}^{0}$ and $\Phi_{8}^{0}$ states on $r$.

We adopt the MATSLISE package to solve the Schrödinger equation with the effective potentials of $\Phi^{ \pm}\left(\Phi^{0}\right), \Phi_{s 1}^{0}$ and $\Phi_{8}^{0}$ states. MATSLISE is a graphical Matlab software package for the numerical study of regular Sturm-Liouville problems, one-dimensional Schrödinger equations and radial Schrödinger equations with a distorted coulomb potential. It allows the fast and accurate computation of the eigenvalues and the visualization of the corresponding eigenfunctions [29].

The binding energies with typical values of $\Lambda$ for $\Phi^{ \pm}\left(\Phi^{0}\right), \Phi_{s 1}^{0}$ and $\Phi_{8}^{0}$ are presented in Table IV, if there exists the solution by solving Schrödinger equation. $r_{\text {rms }}$ denotes the root-mean-square radius with the unit of fm. For $\Phi^{ \pm}\left(\Phi^{0}\right)$, we can not find bound state solutions in the range of $\Lambda<10 \mathrm{GeV}$, which indicates $\Phi^{ \pm}\left(\Phi^{0}\right)$ does not exist. For both $\Phi_{s 1}^{0}$ and $\Phi_{8}^{0}$, there exist bound state solutions with $\Lambda$ around $0.5 \mathrm{GeV}$. When the binding energy $E$ becomes smaller, the $r_{\mathrm{r} m s}$ becomes larger. Similar observations hold for the $B-\bar{B}$ system. Now the reduced masses of $\Omega_{s 1}^{0}$ and $\Omega_{8}^{0}$ are heavier. Hence the kinetic term is smaller. $\Omega_{s 1}^{0}$ and $\Omega_{8}^{0}$ states appear with a larger $\Lambda$ around $1.1 \mathrm{GeV}$. As an example, we show the dependence of the binding energy of $\Phi_{s 1}^{0}$ on $\Lambda$ in Fig. 3. Moreover, we increase and reduce both the vector coupling constant and scalar coupling constant by a factor of two to see the variation of the binding energy and the cutoff parameter. The numerical results are collected in Tables V] and VI]

\section{THE $\mathcal{D}^{*}-\overline{\mathcal{D}}^{*}$ CASE}

\section{A. The potential of the V-V system}

The possible quantum numbers of the $\mathrm{S}$-wave $\mathrm{V}-\mathrm{V}$ system are $J^{P}=1^{+}, 1^{+}, 2^{+}$. The $C$ parity is + for the neutral states. The exchanged mesons include the pseudoscalar, vector and $\sigma$ mesons. The exchange potential reads

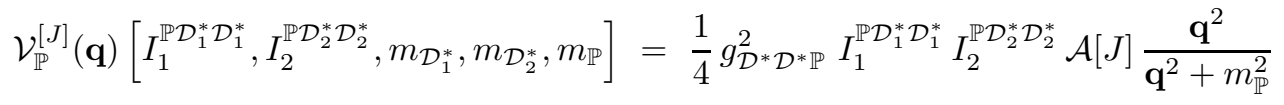




\begin{tabular}{c||ccc}
\hline \hline & & $\mathcal{D}-\overline{\mathcal{D}}$ & $r_{\mathrm{rms}}(\mathrm{fm})$ \\
\hline State & $\Lambda(\mathrm{GeV})$ & $E(\mathrm{MeV})$ & - \\
\hline$\Phi^{ \pm}\left(\Phi^{0}\right)$ & - & - & 1.84 \\
\hline$\Phi_{s 1}^{0}$ & 0.58 & -10.14 & 2.80 \\
& 0.60 & -3.46 & 1.44 \\
\hline$\Phi_{8}^{0}$ & 0.50 & -23.42 & 2.52 \\
& 0.53 & -4.93 & $r_{\mathrm{rms}}(\mathrm{fm})$ \\
\hline & & $\mathcal{B}-\mathcal{B}$ & - \\
\hline State & $\Lambda(\mathrm{GeV})$ & $E(\mathrm{MeV})$ & 1.84 \\
\hline$\Omega^{ \pm}\left(\Omega^{0}\right)$ & - & - & 2.86 \\
\hline$\Omega_{s 1}^{0}$ & 0.70 & -9.24 & 1.95 \\
& 0.72 & -1.78 & 1.23 \\
\hline$\Omega_{8}^{0}$ & 1.10 & -7.13 & -23.34 \\
& 1.15 & & \\
\hline \hline
\end{tabular}

TABLE IV: The bound state solutions for $\Phi^{ \pm}\left(\Phi^{0}\right), \Phi_{s 1}^{0}$ and $\Phi_{8}^{0}$ and $\Omega^{ \pm}\left(\Omega^{0}\right), \Omega_{s 1}^{0}$ and $\Omega_{8}^{0}$.

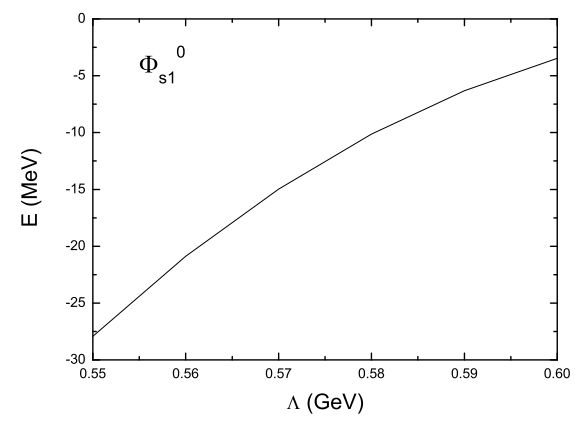

FIG. 3: The dependence of $E$ on $\Lambda$ for $\Phi_{s 1}^{0}$.

for the pseudoscalar meson exchange and

$$
\begin{aligned}
\mathcal{V}_{\mathbb{V}}^{[J]} & (\mathbf{q})\left[I_{1}^{\mathbb{V} \mathcal{D}_{1}^{*} \mathcal{D}_{1}^{*}}, I_{2}^{\mathbb{V} \mathcal{D}_{2}^{*} \mathcal{D}_{2}^{*}}, m_{\mathcal{D}_{1}^{*}}, m_{\mathcal{D}_{2}^{*}}, m_{\mathbb{V}}\right] \\
& =-I_{1}^{\mathbb{V} \mathcal{D}_{1}^{*} \mathcal{D}_{1}^{*}} I_{2}^{\mathbb{V} \mathcal{D}_{2}^{*} \mathcal{D}_{2}^{*}}\left[\frac{g_{\mathcal{D}^{*} \mathcal{D}^{*} \mathbb{V}}^{2}}{4 m_{\mathcal{D}_{1}^{*}} m_{\mathcal{D}_{2}^{*}}} \mathcal{C}[J] \frac{\mathbf{q}^{2}}{m_{\mathbb{V}}^{2}}+g_{\mathcal{D}^{*} \mathcal{D}^{*} \mathbb{V}}^{2} \mathcal{C}[J] \frac{1}{\mathbf{q}^{2}+m_{\mathbb{V}}^{2}}+\frac{4 f_{\mathcal{D}^{*} \mathcal{D}^{*} \mathbb{V}}^{2}}{m_{\mathcal{D}_{1}^{*}} m_{\mathcal{D}_{2}^{*}}} \mathcal{B}[J] \frac{\mathbf{q}^{2}}{\mathbf{q}^{2}+m_{\mathbb{V}}^{2}}\right]
\end{aligned}
$$

\begin{tabular}{c||ccc}
\hline \hline & & $\mathcal{D}-\overline{\mathcal{D}}$ & \\
\hline \multicolumn{1}{c||}{ State } & $\Lambda(\mathrm{GeV})$ & $E(\mathrm{MeV})$ & $r_{\text {rms }}(\mathrm{fm})$ \\
\hline$\Phi^{ \pm}\left(\Phi^{0}\right)$ & - & - & - \\
\hline$\Phi_{s 1}^{0}$ & 0.72 & -23.00 & 1.30 \\
& 0.74 & -8.16 & 1.90 \\
\hline$\Phi_{8}^{0}$ & 0.60 & -37.20 & 1.17 \\
& 0.62 & -11.39 & 1.77 \\
\hline & & $\mathcal{B}-\mathcal{B}$ & $r_{\text {rms }}(\mathrm{fm})$ \\
\hline State & $\Lambda(\mathrm{GeV})$ & $E(\mathrm{MeV})$ & - \\
\hline$\Omega^{ \pm}\left(\Omega^{0}\right)$ & - & - & 1.44 \\
\hline$\Omega_{s 1}^{0}$ & 0.82 & -16.70 & 1.92 \\
\hline$\Omega_{8}^{0}$ & 0.83 & -7.69 & 1.66 \\
& 0.91 & -11.84 & 1.32 \\
\hline \hline
\end{tabular}

TABLE V: The bound state solutions for $\Phi^{ \pm}\left(\Phi^{0}\right), \Phi_{s 1}^{0}$ and $\Phi_{8}^{0}$ and $\Omega^{ \pm}\left(\Omega^{0}\right), \Omega_{s 1}^{0}$ and $\Omega_{8}^{0}$ if we increase the coupling constants by a factor of two. 


\begin{tabular}{c||ccc}
\hline \hline & & $\mathcal{D}-\overline{\mathcal{D}}$ & $r_{\mathrm{rms}}(\mathrm{fm})$ \\
\hline State & $\Lambda(\mathrm{GeV})$ & $E(\mathrm{MeV})$ & - \\
\hline$\Phi^{ \pm}\left(\Phi^{0}\right)$ & - & - & 1.50 \\
\hline$\Phi_{s 1}^{0}$ & 0.38 & -24.49 & 1.63 \\
& 0.39 & -18.89 & 1.78 \\
\hline$\Phi_{8}^{0}$ & 0.38 & -15.38 & 2.02 \\
& 0.39 & -10.61 & $r_{\mathrm{rms}}(\mathrm{fm})$ \\
\hline & & $\mathcal{B}-\mathcal{B}$ & - \\
\hline State & $\Lambda(\mathrm{GeV})$ & $E(\mathrm{MeV})$ & 1.29 \\
\hline$\Omega^{ \pm}\left(\Omega^{0}\right)$ & - & - & 1.55 \\
\hline$\Omega_{s 1}^{0}$ & 0.50 & -30.69 & 1.37 \\
& 0.52 & -17.64 & 1.73 \\
\hline$\Omega_{8}^{0}$ & 0.46 & -29.24 & -14.15 \\
& 0.48 & & \\
\hline \hline
\end{tabular}

TABLE VI: The bound state solutions for $\Phi^{ \pm}\left(\Phi^{0}\right), \Phi_{s 1}^{0}$ and $\Phi_{8}^{0}$ and $\Omega^{ \pm}\left(\Omega^{0}\right), \Omega_{s 1}^{0}$ and $\Omega_{8}^{0}$ if we reduce the coupling constants by a factor of two.

for the vector meson exchange. For the $\sigma$ exchange,

$$
\mathcal{V}_{\sigma}^{[J]}(\mathbf{q})=-g_{\sigma}^{2} \mathcal{C}[J] \frac{1}{\mathbf{q}^{2}+m_{\sigma}^{2}}
$$

with

$$
\begin{aligned}
& \mathcal{A}[J] \equiv \sum_{\lambda_{1} \lambda_{2} \lambda_{3} \lambda_{4}}\left\langle 1 \lambda_{1} ; 1 \lambda_{2} \mid J, m\right\rangle\left\langle 1 \lambda_{3} ; 1 \lambda_{4} \mid J, m\right\rangle \frac{1}{\mathbf{q}^{2}}\left[\epsilon_{1}^{\lambda_{1}} \cdot\left(\mathbf{q} \times \epsilon_{3}^{* \lambda_{3}}\right) \epsilon_{2}^{\lambda_{2}} \cdot\left(\mathbf{q} \times \epsilon_{4}^{* \lambda_{4}}\right)\right], \\
& \mathcal{B}[J] \equiv \sum_{\lambda_{1} \lambda_{2} \lambda_{3} \lambda_{4}}\left\langle 1 \lambda_{1} ; 1 \lambda_{2} \mid J, m\right\rangle\left\langle 1 \lambda_{3} ; 1 \lambda_{4} \mid J, m\right\rangle \frac{1}{\mathbf{q}^{2}}\left[\left(\epsilon_{1} \cdot \mathbf{q}\right)\left(\epsilon_{2} \cdot \mathbf{q}\right)\left(\epsilon_{3}^{*} \cdot \epsilon_{4}^{*}\right)+(c . t . s)\right], \\
& \mathcal{C}[J] \equiv \sum_{\lambda_{1} \lambda_{2} \lambda_{3} \lambda_{4}}\left\langle 1 \lambda_{1} ; 1 \lambda_{2} \mid J, m\right\rangle\left\langle 1 \lambda_{3} ; 1 \lambda_{4} \mid J, m\right\rangle\left(\epsilon_{1}^{\lambda_{1}} \cdot \epsilon_{3}^{* \lambda_{3}}\right)\left(\epsilon_{2}^{\lambda_{2}} \cdot \epsilon_{4}^{* \lambda_{4}}\right),
\end{aligned}
$$

where $\epsilon_{i}$ is the polarization vector, and c.t.s denotes the cross-terms, i.e. $-\left(\epsilon_{1} \cdot \mathbf{q}\right)\left(\epsilon_{4}^{*} \cdot \mathbf{q}\right)\left(\epsilon_{2} \cdot \epsilon_{3}^{*}\right),\left(\epsilon_{3}^{*} \cdot \mathbf{q}\right)\left(\epsilon_{4}^{*} \cdot \mathbf{q}\right)\left(\epsilon_{1} \cdot \epsilon_{2}\right)$ and $-\left(\epsilon_{3}^{*} \cdot \mathbf{q}\right)\left(\epsilon_{2} \cdot \mathbf{q}\right)\left(\epsilon_{1} \cdot \epsilon_{4}^{*}\right)$. When considering the different systems with $J^{P}=0^{+}, 1^{+}, 2^{+}$, we impose the constraint on the scattering amplitudes that the initial states and final states should have the same angular momentum. Then we average the potential in the momentum space, i.e., making the substitutions $q_{x, y, z}^{2} \rightarrow q^{2} / 3$. The coefficients of $\mathcal{A}(J), \mathcal{B}(J)$ and $\mathcal{C}(J)$ are listed into Table VII

\begin{tabular}{c||ccc}
\hline \hline$J$ & $\mathcal{A}(J)$ & $\mathcal{B}(J)$ & $\mathcal{C}(J)$ \\
\hline 0 & $2 / 3$ & $4 / 3$ & 1 \\
1 & $1 / 3$ & $2 / 3$ & 1 \\
2 & $-1 / 3$ & $-2 / 3$ & 1 \\
\hline \hline
\end{tabular}

TABLE VII: The values of $\mathcal{A}(J), \mathcal{B}(J)$ and $\mathcal{C}(J)$ for the cases of $J^{P}=0^{+}, 1^{+}, 2^{+}$systems.

After Fourier transformation, we get the potentials in the coordinate space

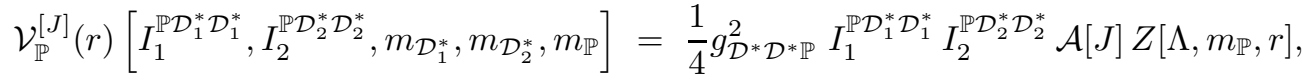

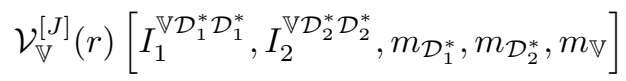

$$
\begin{aligned}
& =-I_{1}^{\mathbb{V} \mathcal{D}_{1}^{*} \mathcal{D}_{1}^{*}} I_{2}^{\mathbb{V} \mathcal{D}_{2}^{*} \mathcal{D}_{2}^{*}}\left[\frac{g_{\mathcal{D}^{*} \mathcal{D}^{*} \mathbb{V}}^{2}}{4 m_{\mathcal{D}_{1}^{*}} m_{\mathcal{D}_{2}^{*}}} \mathcal{C}[J] \frac{1}{m_{\mathbb{V}}^{2}} X\left[\Lambda, m_{\mathbb{V}}, r\right]+g_{\mathcal{D}^{*} \mathcal{D}^{* \mathbb{V}}}^{2} \mathcal{C}[J] Y\left[\Lambda, m_{\mathbb{V}}, r\right]\right. \\
& \left.+\frac{4 f_{\mathcal{D}^{*} \mathcal{D}^{*} \mathbb{V}}^{2}}{m_{\mathcal{D}_{1}^{*}} m_{\mathcal{D}_{2}^{*}}} \mathcal{B}[J] Z\left[\Lambda, m_{\mathbb{V}}, r\right]\right]
\end{aligned}
$$




$$
\mathcal{V}_{\sigma}(r)^{[J]}=-g_{\sigma}^{2} \mathcal{C}[J] Y\left[\Lambda, m_{\sigma}, r\right] .
$$

The total effective potentials for the $\Phi_{s}^{* * \pm}$ and $\Phi_{s}^{0}\left(\bar{\Phi}_{s}^{0}\right)$ states are

$$
\begin{gathered}
\mathcal{V}(r)_{\text {Total }}^{\Phi_{s}^{ \pm}[J]}=\mathcal{V}_{\mathbb{P}}^{[J]}(r)\left[\frac{1}{\sqrt{6}},-\frac{2}{\sqrt{6}}, m_{D^{* 0}}, m_{D_{s}^{*}}, m_{\eta}\right], \\
\mathcal{V}(r)_{\text {Total }}^{\Phi_{s}^{0}\left(\bar{\Phi}_{s}^{0}\right)[J]}=\mathcal{V}_{\mathbb{P}}^{[J]}(r)\left[\frac{1}{\sqrt{6}},-\frac{2}{\sqrt{6}}, m_{D^{*+}}, m_{D_{s}^{*}}, m_{\eta}\right],
\end{gathered}
$$

where only the $\eta$ meson exchange is allowed. Considering the $\mathrm{SU}(2)$ symmetry, one further gets

$$
\mathcal{V}(r)_{\text {Total }}^{\Phi_{s}^{ \pm}[J]} \approx \mathcal{V}(r)_{\text {Total }}^{\Phi_{s}^{0}\left(\bar{\Phi}_{s}^{0}\right)[J]}=-\frac{1}{12} g_{\mathcal{D}^{*} \mathcal{D}^{*} \mathbb{P}}^{2} \mathcal{A}[J] Z\left[\Lambda, m_{\eta}, r\right] .
$$

For the $\Phi_{s 1}^{* * 0}$ state, the potential is

$$
\begin{aligned}
\mathcal{V}(r)_{\text {Total }}^{\Phi_{s 1}^{* * 0}[J]}= & \mathcal{V}_{\mathbb{P}}^{[J]}(r)\left[-\frac{2}{\sqrt{6}},-\frac{2}{\sqrt{6}}, m_{D_{s}^{*}}, m_{D_{s}^{*}}, m_{\eta}\right]+\mathcal{V}_{\mathbb{V}}^{[J]}(r)\left[1,1, m_{D_{s}^{*}}, m_{D_{s}^{*}}, m_{\phi}\right] \\
= & \frac{1}{6} g_{\mathcal{D}^{*} \mathcal{D}^{*} \mathbb{P}}^{2} \mathcal{A}[J] Z\left[\Lambda, m_{\eta}, r\right]-\left[\frac{g_{\mathcal{D}^{*} \mathcal{D} * \mathbb{V}}^{2}}{4 m_{\mathcal{D}_{s}^{*}}^{2} m_{\phi}^{2}} \mathcal{C}[J] X\left[\Lambda, m_{\phi}, r\right]+g_{\mathcal{D}^{*} \mathcal{D} * \mathbb{V}}^{2} \mathcal{C}[J] Y\left[\Lambda, m_{\phi}, r\right]\right. \\
& \left.+\frac{4 f_{\mathcal{D}^{*} \mathcal{D} * \mathbb{V}}^{2}}{m_{\mathcal{D}_{s}^{*}}^{2}} \mathcal{B}[J] Z\left[\Lambda, m_{\phi}, r\right]\right]
\end{aligned}
$$

For $\Phi^{* * \pm}$, the exchange potential reads

$$
\begin{aligned}
\mathcal{V}(r)_{\text {Total }}^{\Phi^{* * \pm}[J]}= & \mathcal{V}_{\mathbb{P}}^{[J]}(r)\left[\frac{1}{\sqrt{2}},-\frac{1}{\sqrt{2}}, m_{D^{* 0}}, m_{D^{*+}}, m_{\pi}\right]+\mathcal{V}_{\mathbb{P}}^{[J]}(r)\left[\frac{1}{\sqrt{6}}, \frac{1}{\sqrt{6}}, m_{D^{* 0}}, m_{D^{*+}}, m_{\eta}\right] \\
& +\mathcal{V}_{\mathbb{V}}^{[J]}(r)\left[\frac{1}{\sqrt{2}},-\frac{1}{\sqrt{2}}, m_{D^{* 0}}, m_{D^{*+}}, m_{\rho}\right]+\mathcal{V}_{\mathbb{V}}^{[J]}(r)\left[\frac{1}{\sqrt{2}}, \frac{1}{\sqrt{2}}, m_{D^{* 0}}, m_{D^{*+}}, m_{\omega}\right] \\
& +\mathcal{V}_{\sigma}(r)^{[J]} \\
\approx & -g_{\mathcal{D}^{*} \mathcal{D}^{*} \mathbb{P}}^{2} \mathcal{A}[J]\left[\frac{Z\left[\Lambda, m_{\pi}, r\right]}{8}-\frac{Z\left[\Lambda, m_{\eta}, r\right]}{24}\right]-g_{\sigma}^{2} \mathcal{C}[J] Y\left[\Lambda, m_{\sigma}, r\right] \\
& +\frac{1}{2}\left[\frac{g_{\mathcal{D}^{*} \mathcal{D}^{*} \mathbb{V}}^{2}}{4 m_{\mathcal{D}^{*}}^{2} m_{\rho}^{2}} \mathcal{C}[J] X\left[\Lambda, m_{\rho}, r\right]+g_{\mathcal{D}^{*} \mathcal{D}^{* \mathbb{V}}}^{2} \mathcal{C}[J] Y\left[\Lambda, m_{\rho}, r\right]+\frac{\left.4 f_{\mathcal{D}^{*} \mathcal{D}^{*} \mathbb{V}}^{2} \mathcal{B}[J] Z\left[\Lambda, m_{\rho}, r\right]\right]}{m_{\mathcal{D}^{*}}^{2}}\right. \\
& -\frac{1}{2}\left[\frac{g_{\mathcal{D}^{*} \mathcal{D}^{*} \mathbb{V}}^{2}}{4 m_{\mathcal{D}^{*}}^{2} m_{\omega}^{2}} \mathcal{C}[J] X\left[\Lambda, m_{\omega}, r\right]+g_{\mathcal{D}^{*} \mathcal{D}^{* \mathbb{V}}}^{2} \mathcal{C}[J] Y\left[\Lambda, m_{\omega}, r\right]+\frac{\left.4 f_{\mathcal{D}^{*} \mathcal{D}^{*} \mathbb{V}}^{2} \mathcal{B}[J] Z\left[\Lambda, m_{\omega}, r\right]\right] .}{m_{\mathcal{D}^{*}}^{2}}\right.
\end{aligned}
$$


The potential for the $\Phi^{* * 0}\left(\Phi_{8}^{* * 0}\right)$ state is

$$
\begin{aligned}
& \mathcal{V}(r)_{\text {Total }}^{\Phi_{8}^{* * 0}\left(\Phi_{8}^{* * 0}\right)[J]}=\frac{1}{2}\left\{\mathcal{V}_{\mathbb{P}}^{[J]}(r)\left[\frac{1}{\sqrt{2}}, \frac{1}{\sqrt{2}}, m_{D^{* 0}}, m_{D^{* 0}}, m_{\pi^{0}}\right] \mp 2 \mathcal{V}_{\mathbb{P}}^{[J]}(r)\left[1,1, m_{D^{* 0}}, m_{D^{*+}}, m_{\pi^{ \pm}}\right]\right. \\
& +\mathcal{V}_{\mathbb{P}}^{[J]}(r)\left[-\frac{1}{\sqrt{2}},-\frac{1}{\sqrt{2}}, m_{D^{*+}}, m_{D^{*-}}, m_{\pi^{0}}\right]+\mathcal{V}_{\mathbb{P}}^{[J]}(r)\left[\frac{1}{\sqrt{6}}, \frac{1}{\sqrt{6}}, m_{D^{* 0}}, m_{D^{* 0}}, m_{\eta}\right] \\
& +\mathcal{V}_{\mathbb{P}}^{[J]}(r)\left[\frac{1}{\sqrt{6}}, \frac{1}{\sqrt{6}}, m_{D^{*+}}, m_{D^{*-}}, m_{\eta}\right] \\
& +\mathcal{V}_{\mathbb{V}}^{[J]}(r)\left[\frac{1}{\sqrt{2}}, \frac{1}{\sqrt{2}}, m_{D^{* 0}}, m_{D^{* 0}}, m_{\rho^{0}}\right] \mp 2 \mathcal{V}_{\mathbb{V}}^{[J]}(r)\left[1,1, m_{D^{* 0}}, m_{D^{*+}}, m_{\rho^{ \pm}}\right] \\
& +\mathcal{V}_{\mathbb{V}}^{[J]}(r)\left[-\frac{1}{\sqrt{2}},-\frac{1}{\sqrt{2}}, m_{D^{*+}}, m_{D^{*-}}, m_{\rho^{0}}\right]+\mathcal{V}_{\mathbb{V}}^{[J]}(r)\left[\frac{1}{\sqrt{2}}, \frac{1}{\sqrt{2}}, m_{D^{* 0}}, m_{D^{* 0}}, m_{\omega}\right] \\
& \left.+\mathcal{V}_{\mathbb{V}}^{[J]}(r)\left[\frac{1}{\sqrt{2}}, \frac{1}{\sqrt{2}}, m_{D^{*+}}, m_{D^{*-}}, m_{\omega}\right]+2 \mathcal{V}_{\sigma}^{[J]}(r)\right\} \\
& \approx g_{\mathcal{D}^{*} \mathcal{D}^{*} \mathbb{P}}^{2} \mathcal{A}[J]\left[\frac{(1 \mp 2)}{8} Z\left[\Lambda, m_{\pi}, r\right]+\frac{Z\left[\Lambda, m_{\eta}, r\right]}{24}\right]-g_{\sigma}^{2} \mathcal{C}[J] Y\left[\Lambda, m_{\sigma}, r\right] \\
& -\frac{(1 \mp 2)}{2}\left[\frac{g_{\mathcal{D}^{*} \mathcal{D}^{*} \mathbb{V}}^{2}}{4 m_{\mathcal{D}^{*}}^{2} m_{\rho}^{2}} \mathcal{C}[J] X\left[\Lambda, m_{\rho}, r\right]+g_{\mathcal{D}^{*} \mathcal{D}^{*} \mathbb{V}}^{2} \mathcal{C}[J] Y\left[\Lambda, m_{\rho}, r\right]+\frac{4 f_{\mathcal{D}^{*} \mathcal{D}^{*} \mathbb{V}}^{2}}{m_{\mathcal{D}^{*}}^{2}} \mathcal{B}[J] Z\left[\Lambda, m_{\rho}, r\right]\right] \\
& -\frac{1}{2}\left[\frac{g_{\mathcal{D}^{*} \mathcal{D}^{* \mathbb{V}}}^{2}}{4 m_{\mathcal{D}^{*}}^{2} m_{\omega}^{2}} \mathcal{C}[J] X\left[\Lambda, m_{\omega}, r\right]+g_{\mathcal{D}^{*} \mathcal{D}^{* \mathbb{V}}}^{2} \mathcal{C}[J] Y\left[\Lambda, m_{\omega}, r\right]+\frac{4 f_{\mathcal{D}^{*} \mathcal{D}^{*} \mathbb{V}}^{2}}{m_{\mathcal{D}^{*}}^{2}} \mathcal{B}[J] Z\left[\Lambda, m_{\omega}, r\right]\right],
\end{aligned}
$$

where $\mp$ corresponds to $\Phi^{* * 0}\left(\Phi_{8}^{* * 0}\right)$ respectively. As a cross-check, the potential for $\Phi^{* * 0}$ is the same as that for $\Phi^{* * \pm}$ from the SU(2) symmetry. For the V-V systems, we need only consider four independent types of potentials corresponding to $\left[\Phi_{s}^{* * \pm}, \Phi_{s}^{* * 0}, \bar{\Phi}_{s}^{* * 0}\right],\left[\Phi^{* * \pm}, \Phi^{* * 0}\right], \Phi_{8}^{* * 0}$ and $\Phi_{s 1}^{* * 0}$ listed in Eqs. (32), (34), (35), (33) respectively when we consider the $\mathrm{SU}(2)$ symmetry. For states with different quantum numbers, we simply change the values of $\mathcal{A}[J]$, $\mathcal{B}[J]$ and $\mathcal{C}[J]$ in Table VII]

\section{B. Numerical results for the V-V system}

The quantum numbers of the S-wave $\mathrm{V}-\mathrm{V}$ system are $J^{P}=0^{+}, 1^{+}, 2^{+}$. The variation of the oscillating effective potential of $\Phi_{s}^{* * \pm}\left(\Phi_{s}^{* * 0}, \bar{\Phi}_{s}^{* * 0}\right)$ with $r$ is shown in Fig. 4, where only the $\eta$ meson exchange is allowed. The effective potentials of $\Phi^{* \pm}\left(\Phi^{* 0}\right)$ and $\Phi_{8}^{* * 0}$ with $J^{P}=0^{+}, 1^{+}, 2^{+}$are presented in Fig. [5]6 where the exchanged mesons include $\pi, \eta, \rho, \omega$ and $\sigma$. For $\Phi_{s 1}^{* * 0}$, its effective potential is shown in Fig. 7, where both the $\eta$ and $\phi$ meson exchange is allowed.

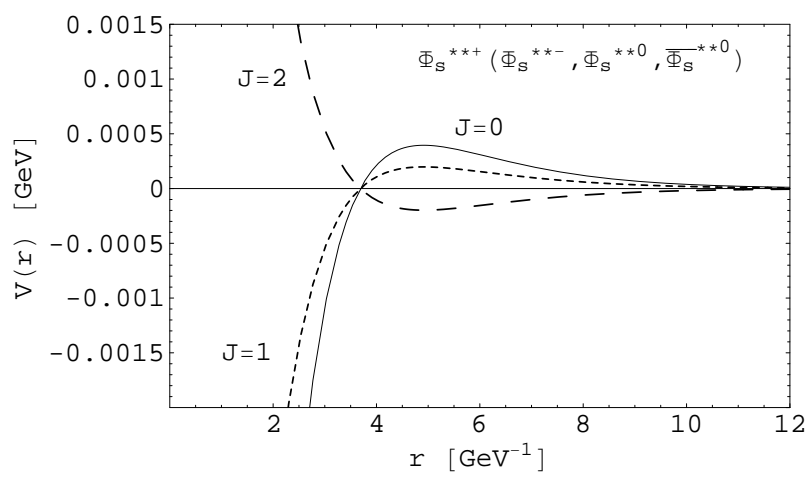

FIG. 4: The effective potential of $\Phi_{s}^{* * \pm}\left(\Phi_{s}^{* * 0}, \bar{\Phi}_{s}^{* * 0}\right)$. 


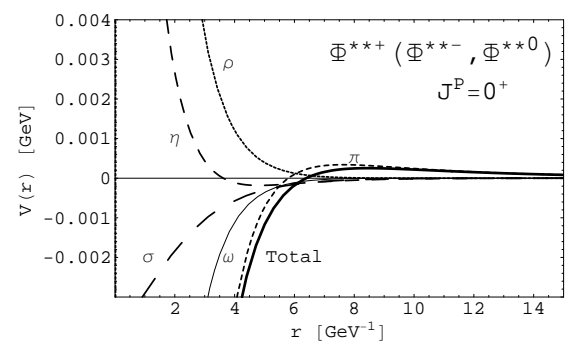

(a)

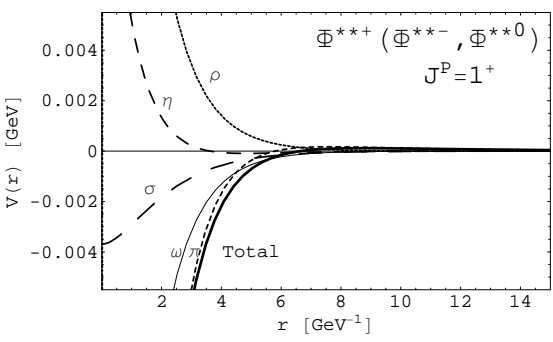

(b)

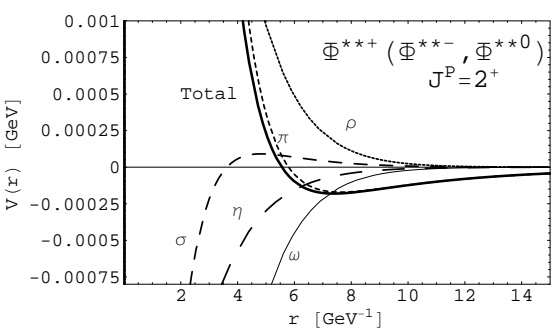

(c)

FIG. 5: The effective potentials of $\Phi^{* * \pm}\left(\Phi^{* * 0}\right)$ with different quantum numbers. The thick solid line is the total effective potential.

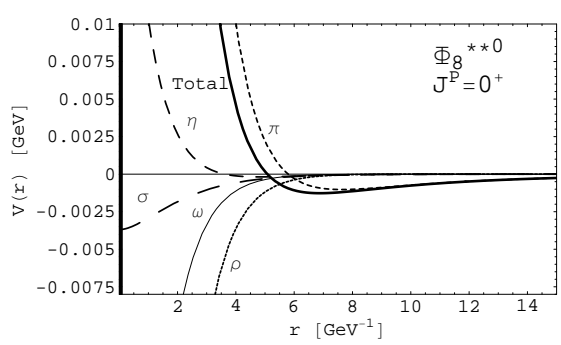

(a)

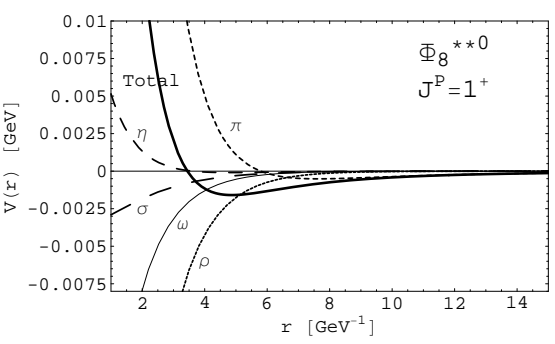

(b)

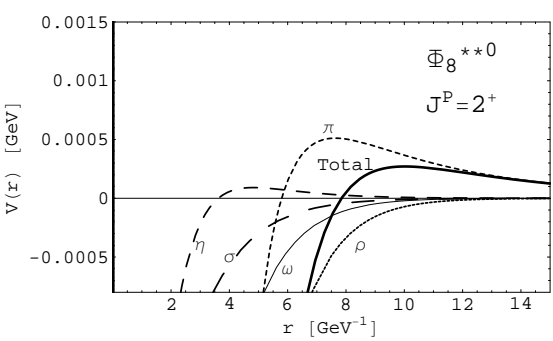

(c)

FIG. 6: The shape of the exchange potential of $\Phi_{8}^{* * 0}$. The thick solid line is the total effective potential.

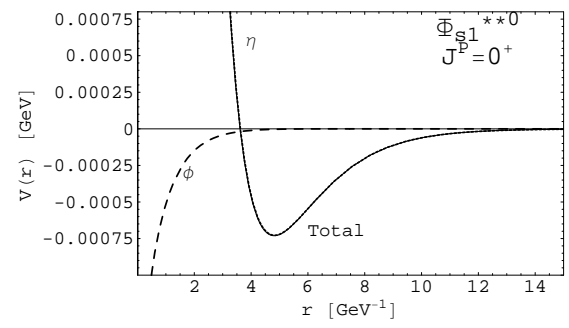

(a)

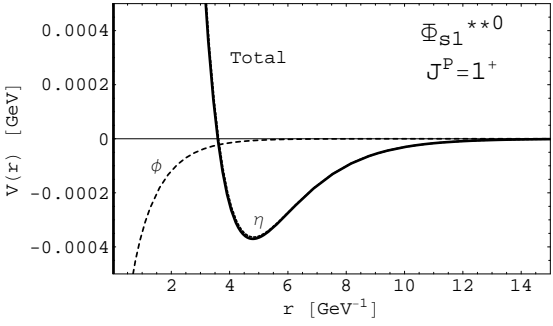

(b)

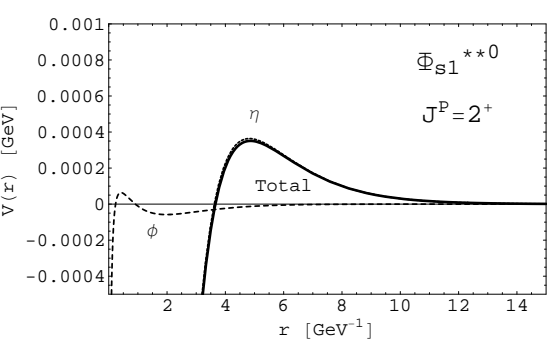

(c)

FIG. 7: The variation of the effective potential of $\Phi_{s 1}^{* * 0}$ with $r$. The thick solid line is the total effective potential.

With the above effective potentials, we solve the Schrödinger equation to find the bound state solutions for the V-V system. Numerical results are collected in Table VIII. For the $\mathcal{D}^{*}-\mathcal{D}^{*}$ states with $J^{P}=0^{+}, 1^{+}$, a bound state exists only for $\Lambda$ much larger than $1 \mathrm{GeV}$. Especially for $\Phi_{s}^{* * \pm}\left(\Phi_{s}^{* * 0}, \bar{\Phi}_{s}^{* * 0}\right)$ and $\Phi^{* * \pm}\left(\Phi^{* * 0}\right)$ with $J^{P}=1^{+}$, the existence of a bound state requires the values of $\Lambda$ be larger than $8 \mathrm{GeV}$. For $\Phi_{s}^{* * \pm}\left(\Phi_{s}^{* * 0}, \bar{\Phi}_{s}^{* * 0}\right)$ and $\Phi^{* * \pm}\left(\Phi^{* * 0}\right)$ with $J^{P}=2^{+}$, no bound states exists for $\Lambda<10 \mathrm{GeV}$. The cutoff parameter $\Lambda$ is a typical hadronic scale, which is generally expected to be around $1 \sim 2 \mathrm{GeV}$. If $\Lambda$ is much larger than $2 \mathrm{GeV}$ or much smaller than $1 \mathrm{GeV}$ in order to form a bound state, we tend to conclude that there does not exist a heavy molecular state for these systems. We find bound state solutions for $\Phi_{8}^{* * 0}$ and $\Phi_{s 1}^{* * 0}$ with $J^{P}=0^{+}, 1^{+}, 2^{+}$.

In Table VIII we also give the numerical results for the $\mathcal{B}^{*}-\overline{\mathcal{B}}^{*}$ system. There do not exist molecular states $\Omega_{s}^{* * \pm}\left(\Omega_{s}^{* * 0}, \bar{\Omega}_{s}^{* * 0}\right)$ and $\Omega^{* * \pm}\left(\Omega^{* * 0}\right)$ with $J^{P}=2^{+}$since we can not find bound state solutions for $\Lambda=0 \sim 10 \mathrm{GeV}$. Different from the $\mathcal{D}^{*}-\overline{\mathcal{D}}^{*}$ system, there exist possible molecular states $\Omega_{s}^{* * \pm}\left(\Omega_{s}^{* * 0}, \bar{\Omega}_{s}^{* * 0}\right)$ with $J^{P}=0^{+}, 1^{+}$with more reasonable $\Lambda$ close to $1 \mathrm{GeV}$. We also find the bound state solutions for $\Omega_{8}^{* 0}$ and $\Omega_{s 1}^{* * 0}$. The reason is simple. The larger the reduced mass for $\mathcal{B}^{*}-\overline{\mathcal{B}}^{*}$, the lower the kinetic energy, the easier to form a molecular state. With $\Phi_{s}^{* *}$ as an example, we illustrate the dependence of $E$ on $\Lambda$ in Fig. 8 . When all coupling constants are enlarged by a 
factor of two, the numerical results are collected in Table IX. When all coupling constants are reduced by a factor of two, the results are collected in Table $\mathrm{X}$

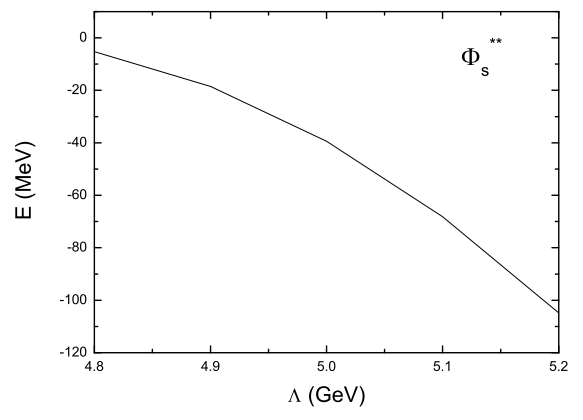

FIG. 8: The dependence of binding energy $E$ on $\Lambda$ for $\Phi_{s}^{* *}$.

\begin{tabular}{|c|c|c|c|c|c|c|c|c|c|}
\hline & \multicolumn{9}{|c|}{$\overline{\mathcal{D}^{*}-\overline{\mathcal{D}}^{*}}$} \\
\hline & \multicolumn{3}{|c|}{$J^{P}=0^{+}$} & \multicolumn{3}{|c|}{$J^{P}=1^{+}$} & \multicolumn{3}{|c|}{$J^{P}=2^{+}$} \\
\hline State & $\Lambda(\mathrm{GeV})$ & $E(\mathrm{MeV})$ & $r_{\mathrm{rms}}(\mathrm{fm})$ & $\Lambda(\mathrm{GeV})$ & $E(\mathrm{MeV})$ & $r_{\mathrm{rms}}(\mathrm{fm})$ & $\Lambda(\mathrm{GeV})$ & $E(\mathrm{MeV})$ & $r_{\mathrm{rms}}(\mathrm{fm})$ \\
\hline \multirow{2}{*}{$\Phi_{s}^{* * \pm}\left(\Phi_{s}^{* * 0}, \Phi_{s}^{* * 0}\right)$} & 4.80 & -5.26 & 1.92 & 8.70 & -1.60 & 2.56 & - & - & - \\
\hline & 5.00 & -39.42 & 0.72 & 8.90 & -21.21 & 0.96 & - & - & - \\
\hline \multirow[t]{2}{*}{$\Phi^{* * \pm}\left(\Phi^{* * 0}\right)$} & 4.00 & -5.21 & 1.99 & 9.80 & -8.40 & 1.61 & - & - & - \\
\hline & 4.20 & -19.02 & 1.08 & 10.00 & -16.53 & 1.19 & - & - & - \\
\hline \multirow[t]{2}{*}{$\Phi_{8}^{* * 0}$} & 0.52 & -18.32 & 1.48 & 0.50 & -37.61 & 1.18 & 0.52 & -11.71 & 1.85 \\
\hline & 0.53 & -8.61 & 1.95 & 0.52 & -15.03 & 1.63 & 0.50 & -24.01 & 1.47 \\
\hline \multirow{4}{*}{$\Phi_{s 1}^{* * 0}$} & 0.63 & -12.91 & 1.55 & 0.60 & -17.27 & 1.45 & 0.54 & -23.64 & 1.46 \\
\hline & 0.64 & -7.13 & 1.93 & 0.62 & -6.56 & 2.11 & 0.56 & -13.01 & 1.77 \\
\hline & \multicolumn{9}{|c|}{$\mathcal{B}^{*}-\mathcal{B}^{*}$} \\
\hline & \multicolumn{3}{|c|}{$J^{P}=0^{+}$} & \multicolumn{3}{|c|}{$J^{P}=1^{+}$} & \multicolumn{3}{|c|}{$J^{P}=2^{+}$} \\
\hline State & $\overline{\Lambda(\mathrm{GeV})}$ & $\overline{E(\mathrm{MeV})}$ & $\overline{r_{\mathrm{rms}}(\mathrm{fm})}$ & $\overline{\Lambda(\mathrm{GeV})}$ & $\overline{E(\mathrm{MeV})}$ & $r_{\mathrm{rms}}(\mathrm{fm})$ & $\Lambda(\mathrm{GeV})$ & $\overline{E(\mathrm{MeV})}$ & $\overline{r_{\mathrm{rms}}(\mathrm{fm})}$ \\
\hline \multirow{2}{*}{$\Omega_{s}^{* * \pm}\left(\Omega_{s}^{* * 0}, \Omega_{s}^{* * 0}\right)$} & 2.30 & -0.57 & 4.76 & 3.80 & -3.98 & 2.21 & - & - & - \\
\hline & 2.40 & -14.90 & 1.17 & 3.90 & -17.91 & 1.06 & - & - & - \\
\hline \multirow[t]{2}{*}{$\Omega^{* * \pm}\left(\Omega^{* * 0}\right)$} & 1.50 & -10.17 & 1.53 & 2.70 & -3.60 & 2.41 & - & - & - \\
\hline & 1.60 & -21.75 & 1.10 & 2.90 & -15.55 & 1.22 & - & - & - \\
\hline \multirow[t]{2}{*}{$\overline{\Omega_{8}^{* * 0}}$} & 0.57 & -23.16 & 1.36 & 0.57 & -32.70 & 1.23 & 0.61 & -18.07 & 1.49 \\
\hline & 0.58 & -6.42 & 2.28 & 0.59 & -5.65 & 2.39 & 0.63 & -8.39 & 1.93 \\
\hline \multirow[t]{2}{*}{$\Omega_{s 1}^{* * 0}$} & 0.74 & -12.12 & 1.56 & 0.70 & -33.5 & 1.09 & 0.64 & -35.37 & 1.25 \\
\hline & 0.75 & -4.48 & 2.37 & 0.72 & -12.68 & 1.58 & 0.65 & -27.12 & 1.35 \\
\hline
\end{tabular}

TABLE VIII: The numerical results for the $\mathcal{D}^{*}-\overline{\mathcal{D}}^{*}$ and $\mathcal{B}^{*}-\overline{\mathcal{B}}^{*}$ systems.

\section{THE $\mathcal{D}^{*}-\overline{\mathcal{D}}$ CASE}

\section{A. The potential of the P-V system}

Fig. 9 shows the the general scattering channels in the derivation of the exchange potential. Different from the $\mathrm{P}-\mathrm{P}$ and $\mathrm{V}-\mathrm{V}$ systems, we need consider two additional crossed scattering diagrams for the P-V system, i.e. Fig. 9 (b) and (c). 


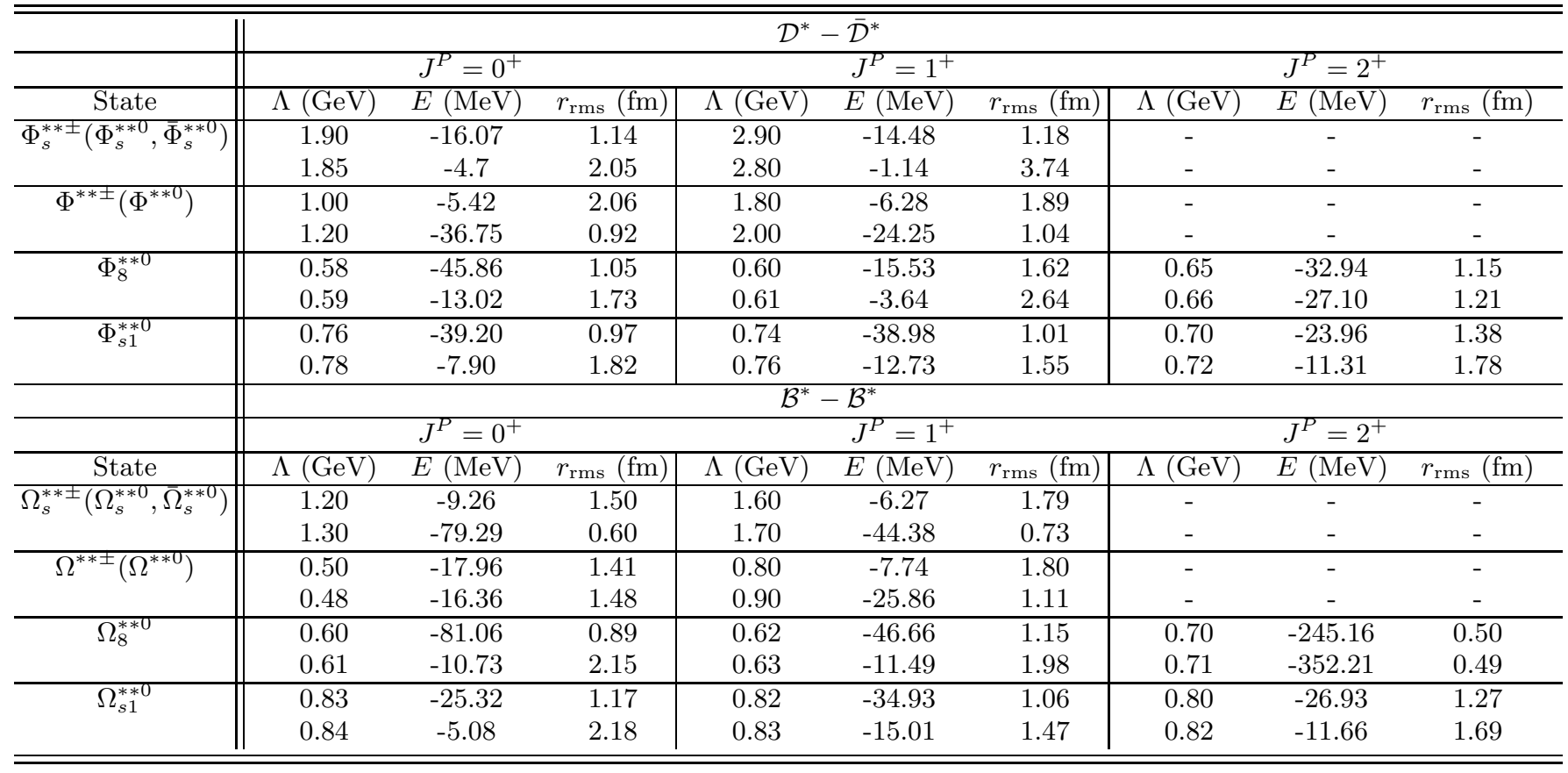

TABLE IX: The numerical results for the $\mathcal{D}^{*}-\overline{\mathcal{D}}^{*}$ and $\mathcal{B}^{*}-\overline{\mathcal{B}}^{*}$ systems after increasing the coupling constants by a factor of two.

\begin{tabular}{|c|c|c|c|c|c|c|c|c|c|}
\hline & \multicolumn{9}{|c|}{$\mathcal{D}^{*}-\overline{\mathcal{D}}^{*}$} \\
\hline & \multicolumn{3}{|c|}{$J^{P}=0^{+}$} & \multicolumn{3}{|c|}{$J^{P}=1^{+}$} & \multicolumn{3}{|c|}{$J^{P}=2^{+}$} \\
\hline State & $\Lambda(\mathrm{GeV})$ & $E(\mathrm{MeV})$ & $r_{\mathrm{rms}}(\mathrm{fm})$ & $\Lambda(\mathrm{GeV})$ & $E(\mathrm{MeV})$ & $r_{\mathrm{rms}}(\mathrm{fm})$ & $\Lambda(\mathrm{GeV})$ & $E(\mathrm{MeV})$ & $r_{\mathrm{rms}}(\mathrm{fm})$ \\
\hline$\Phi_{s}^{* * \pm}\left(\Phi_{s}^{* * 0}, \bar{\Phi}_{s}^{* * 0}\right)$ & - & - & - & - & - & - & - & - & - \\
\hline$\Phi^{* * \pm}\left(\Phi^{* * 0}\right)$ & - & - & - & - & - & - & - & - & - \\
\hline$\Phi_{8}^{* * 0}$ & $\begin{array}{l}0.40 \\
0.41\end{array}$ & $\begin{array}{l}-18.40 \\
-12.23\end{array}$ & $\begin{array}{l}1.57 \\
1.81\end{array}$ & $\begin{array}{l}0.40 \\
0.41\end{array}$ & $\begin{array}{c}-13.09 \\
-8.43\end{array}$ & $\begin{array}{l}1.82 \\
2.12\end{array}$ & $\begin{array}{l}0.39 \\
0.40\end{array}$ & $\begin{array}{c}-10.14 \\
-6.67\end{array}$ & $\begin{array}{l}2.10 \\
2.42\end{array}$ \\
\hline$\Phi_{s 1}^{* * \sigma}$ & $\begin{array}{l}0.46 \\
0.47\end{array}$ & $\begin{array}{l}-6.43 \\
-3.70\end{array}$ & $\begin{array}{l}2.21 \\
2.76\end{array}$ & $\begin{array}{l}0.40 \\
0.42\end{array}$ & $\begin{array}{l}-27.87 \\
-16.15\end{array}$ & $\begin{array}{l}1.37 \\
1.63\end{array}$ & $\begin{array}{l}0.40 \\
0.41\end{array}$ & $\begin{array}{c}-12.05 \\
-8.61\end{array}$ & $\begin{array}{l}1.95 \\
2.18\end{array}$ \\
\hline & \multicolumn{9}{|c|}{$\mathcal{B}^{*}-\mathcal{B}^{*}$} \\
\hline & & $J^{P}=0^{+}$ & & & $J^{P}=1^{+}$ & & & $J^{P}=2^{+}$ & \\
\hline State & $\Lambda(\mathrm{GeV})$ & $E(\mathrm{MeV})$ & $r_{\mathrm{rms}}(\mathrm{fm})$ & $\Lambda(\mathrm{GeV})$ & $E(\mathrm{MeV})$ & $r_{\text {rms }}(\mathrm{fm})$ & $\Lambda(\mathrm{GeV})$ & $E(\mathrm{MeV})$ & $r_{\mathrm{rms}}(\mathrm{fm})$ \\
\hline$\Omega_{s}^{* * \pm}\left(\Omega_{s}^{* * 0}, \Omega_{s}^{* * 0}\right)$ & $\begin{array}{l}6.80 \\
6.90\end{array}$ & $\begin{array}{c}-9.33 \\
-23.17\end{array}$ & $\begin{array}{l}1.43 \\
0.93\end{array}$ & $\begin{array}{l}- \\
-\end{array}$ & $\begin{array}{l}- \\
-\end{array}$ & $\begin{array}{l}- \\
-\end{array}$ & $\begin{array}{l}- \\
-\end{array}$ & $\begin{array}{l}- \\
-\end{array}$ & $\begin{array}{l}- \\
-\end{array}$ \\
\hline$\Omega^{* * \pm}\left(\Omega^{* * 0}\right)$ & $\begin{array}{l}6.10 \\
6.20\end{array}$ & $\begin{array}{c}-7.37 \\
-14.64\end{array}$ & $\begin{array}{l}1.64 \\
1.20\end{array}$ & - & $\begin{array}{l}- \\
-\end{array}$ & $\begin{array}{l}- \\
-\end{array}$ & $\begin{array}{l}- \\
-\end{array}$ & - & $\begin{array}{l}- \\
-\end{array}$ \\
\hline$\Omega_{8}^{* * 0}$ & $\begin{array}{l}0.48 \\
0.50\end{array}$ & $\begin{array}{c}-26.88 \\
-8.85\end{array}$ & $\begin{array}{l}1.31 \\
1.97\end{array}$ & $\begin{array}{l}0.48 \\
0.50\end{array}$ & $\begin{array}{c}-19.59 \\
-6.57\end{array}$ & $\begin{array}{l}1.51 \\
2.25\end{array}$ & $\begin{array}{l}0.48 \\
0.50\end{array}$ & $\begin{array}{c}-11.34 \\
-4.18\end{array}$ & $\begin{array}{l}1.92 \\
2.75\end{array}$ \\
\hline$\Omega_{s 1}^{* * \sigma}$ & $\begin{array}{l}0.58 \\
0.60\end{array}$ & $\begin{array}{l}-11.18 \\
-3.11\end{array}$ & $\begin{array}{l}1.68 \\
2.85\end{array}$ & $\begin{array}{l}0.53 \\
0.55\end{array}$ & $\begin{array}{l}-29.28 \\
-15.49\end{array}$ & $\begin{array}{l}1.23 \\
1.54\end{array}$ & $\begin{array}{l}0.50 \\
0.52\end{array}$ & $\begin{array}{c}-18.73 \\
-9.95\end{array}$ & $\begin{array}{l}1.60 \\
1.97\end{array}$ \\
\hline
\end{tabular}

TABLE X: The numerical results for the $\mathcal{D}^{*}-\overline{\mathcal{D}}^{*}$ and $\mathcal{B}^{*}-\overline{\mathcal{B}}^{*}$ systems after reducing the coupling constants by a factor of two.

In terms of the effective Lagrangian in Sec. III] the $\sigma$ meson exchange potential in the momentum space is [31]

$$
\mathcal{V}_{\sigma}(\mathbf{q})=-\frac{g_{\sigma}^{2}}{\mathbf{q}^{2}+m_{\sigma}^{2}}
$$




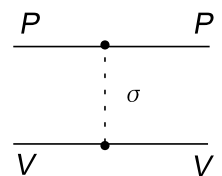

(a)

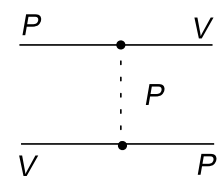

(b)

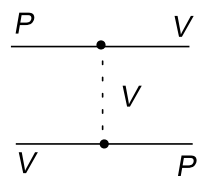

(c)

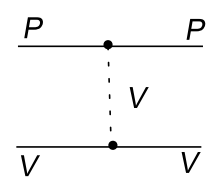

(d)

FIG. 9: The diagrams in the derivation of the effective potential of the P-V system.

The pseudoscalar meson exchange potential reads [15]

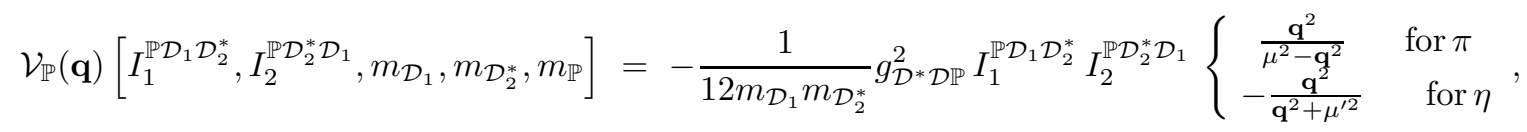

where $\mu=\sqrt{q_{0}^{2}-m_{\pi}^{2}}, \mu^{\prime}=\sqrt{m_{\eta(K)}^{2}-q_{0}^{2}}$ and $q_{0}=m_{D_{2}^{*}}-m_{D_{1}}$. For the vector meson exchange, there exist two types of potentials from the direct and crossed scattering channels:

$$
\begin{gathered}
\mathcal{V}_{\mathbb{V}}^{\text {Direct }}(\mathbf{q})\left[I_{1}^{\mathbb{V} \mathcal{D}_{1} \mathcal{D}_{1}}, I_{2}^{\mathbb{V} \mathcal{D}_{2}^{*} \mathcal{D}_{2}^{*}}, m_{\mathcal{D}_{1}}, m_{\mathcal{D}_{2}^{*}}, m_{\mathbb{V}}\right]=-g_{\mathcal{D} \mathcal{D} \mathbb{V}} g_{\mathcal{D}^{*} \mathcal{D}^{*} \mathbb{V}} I_{1}^{\mathbb{V} \mathcal{D}_{1} \mathcal{D}_{1}} I_{2}^{\mathbb{V} \mathcal{D}_{2}^{*} \mathcal{D}_{2}^{*}}\left(\frac{\mathbf{q}^{2}}{4 m_{D_{1}} m_{D_{2}^{*}} m_{\mathbb{V}}^{2}}+\frac{1}{\mathbf{q}^{2}+m_{\mathbb{V}}^{2}}\right), \\
\mathcal{V}_{\mathbb{V}}^{\text {Cross }}(\mathbf{q})\left[I_{1}^{\mathbb{V} \mathcal{D}_{1} \mathcal{D}_{2}^{*}}, I_{2}^{\mathbb{V} \mathcal{D}_{2}^{*} \mathcal{D}_{1}}, m_{\mathcal{D}_{1}}, m_{\mathcal{D}_{2}^{*}}, m_{\mathbb{V}}\right]=-\frac{8}{3} f_{\mathcal{D}^{*} \mathcal{D} \mathbb{V}}^{2} I_{1}^{\mathbb{V} \mathcal{D}_{1} \mathcal{D}_{2}^{*}} I_{2}^{\mathbb{V} \mathcal{D}_{2}^{*} \mathcal{D}_{1}} \frac{\mathbf{q}^{2}}{\mathbf{q}^{2}+\zeta^{2}},
\end{gathered}
$$

where $\zeta_{\mathcal{V}}=\sqrt{m_{\mathcal{V}}^{2}-q_{0}^{2}}$. One notes that $m_{\mathcal{V}}$ is larger than $q_{0}$ for the P-V case. After Fourier transformation, we obtain the effective potentials in the coordinate space

$$
\begin{aligned}
& \mathcal{V}_{\sigma}(r)=-g_{\sigma}^{2} Y\left[\Lambda, m_{\sigma}, r\right], \\
& \mathcal{V}_{\mathbb{P}}(r)\left[I_{1}^{\mathbb{P} \mathcal{D}_{1} \mathcal{D}_{2}^{*}}, I_{2}^{\mathbb{P} \mathcal{D}_{2}^{*} \mathcal{D}_{1}}, m_{\mathcal{D}_{1}}, m_{\mathcal{D}_{2}^{*}}, m_{\mathbb{P}}\right]=-\frac{1}{12 m_{\mathcal{D}_{1}} m_{\mathcal{D}_{2}^{*}}} g_{\mathcal{D}^{*} \mathcal{D P}}^{2} I_{1}^{\mathbb{P} \mathcal{D}_{1} \mathcal{D}_{2}^{*}} I_{2}^{\mathbb{P} \mathcal{D}_{2}^{*} \mathcal{D}_{1}}\left\{\begin{array}{cc}
U(r) & \text { for } \pi \\
-Z\left[\Lambda, \mu^{\prime}, r\right] & \text { for } \eta
\end{array},\right. \\
& \mathcal{V}_{\mathbb{V}}^{\text {Direct }}(r)\left[I_{1}^{\mathbb{V} \mathcal{D}_{1} \mathcal{D}_{1}}, I_{2}^{\mathbb{V} \mathcal{D}_{2}^{*} \mathcal{D}_{2}^{*}}, m_{\mathcal{D}_{1}}, m_{\mathcal{D}_{2}^{*}}, m_{\mathbb{V}}\right]=-g_{\mathcal{D} \mathcal{D} \mathbb{V}} g_{\mathcal{D}^{*} \mathcal{D}^{*} \mathbb{V}} I_{1}^{\mathbb{V} \mathcal{D}_{1} \mathcal{D}_{1}} I_{2}^{\mathbb{V} \mathcal{D}_{2}^{*} \mathcal{D}_{2}^{*}}\left(\frac{X\left[\Lambda, m_{\mathbb{V}}, r\right]}{4 m_{D_{1}} m_{D_{2}^{*}} m_{\mathbb{V}}^{2}}+Y\left[\Lambda, m_{\mathbb{V}}, r\right]\right), \\
& \mathcal{V}_{\mathbb{V}}^{\text {Cross }}(r)\left[I_{1}^{\mathbb{V} \mathcal{D}_{1} \mathcal{D}_{2}^{*}}, I_{2}^{\mathbb{V} \mathcal{D}_{2}^{*} \mathcal{D}_{1}}, m_{\mathcal{D}_{1}}, m_{\mathcal{D}_{2}^{*}}, m_{\mathbb{V}}\right]=-\frac{8}{3} f_{\mathcal{D}^{*} \mathcal{D} \mathbb{V}}^{2} I_{1}^{\mathbb{V} \mathcal{D}_{1} \mathcal{D}_{2}^{*}} I_{2}^{\mathbb{V} \mathcal{D}_{2}^{*} \mathcal{D}_{1}} Z[\Lambda, \zeta \mathcal{V}, r]
\end{aligned}
$$

Here

$$
U(r)=-\frac{\mu^{2}}{4 \pi r}\left[\cos (\mu r)-e^{-\alpha r}\right]-\frac{\beta^{2} \alpha}{8 \pi} e^{-\alpha r}
$$

with $\beta=\sqrt{\Lambda^{2}-m_{\pi}^{2}}$ and $\alpha=\sqrt{\Lambda^{2}-q_{0}^{2}}$. The total effective potentials of $\Phi_{s}^{* \pm} / \hat{\Phi}_{s}^{* \pm}, \Phi_{s}^{* 0} / \hat{\Phi}_{s}^{* 0}, \bar{\Phi}_{s}^{* 0} / \hat{\bar{\Phi}}_{s}^{* 0}$

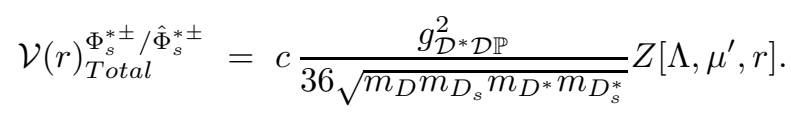

For $\Phi^{* \pm} / \hat{\Phi}^{* \pm}$, the total effective potential reads

$$
\begin{aligned}
& \mathcal{V}(r)_{\text {Total }}^{\Phi^{* \pm} / \hat{\Phi}^{* \pm}}=\frac{1}{2} g_{\mathcal{D D} \mathbb{V}} g_{\mathcal{D}^{*} \mathcal{D}^{*} \mathbb{V}}\left[\frac{X\left[\Lambda, m_{\rho}, r\right]}{4 m_{D} m_{D^{*}} m_{\rho}^{2}}+Y\left[\Lambda, m_{\rho}, r\right]\right]-\frac{1}{2} g_{\mathcal{D D D} \mathbb{V}} g_{\mathcal{D}^{*} \mathcal{D}^{*} \mathbb{V}}\left[\frac{X\left[\Lambda, m_{\omega}, r\right]}{4 m_{D} m_{D^{*}} m_{\omega}^{2}}+Y\left[\Lambda, m_{\omega}, r\right]\right] \\
& -c\left\{-\frac{g_{\mathcal{D}^{*} \mathcal{D P}}^{2}}{24 m_{D} m_{D^{*}}} U(r)-\frac{g_{\mathcal{D}^{*} \mathcal{D P}}^{2}}{72 m_{D} m_{D^{*}}} Z\left[\Lambda, \mu^{\prime}, r\right]-\frac{4}{3} f_{\mathcal{D}^{*} \mathcal{D V}}^{2} Z\left[\Lambda, \zeta_{\rho}, r\right]+\frac{4}{3} f_{\mathcal{D}^{*} \mathcal{D} \mathbb{V}}^{2} Z\left[\Lambda, \zeta_{\omega}, r\right]\right\} \\
& -g_{\sigma}^{2} Y\left[\Lambda, m_{\sigma}, r\right] \text {. }
\end{aligned}
$$


For $\Phi^{* 0} / \hat{\Phi}^{* 0}, \Phi_{s 1}^{* 0} / \hat{\Phi}_{s 1}^{* 0}$ and $\Phi_{8}^{* 0} / \hat{\Phi}_{8}^{* 0}$, we have

$$
\begin{aligned}
& \mathcal{V}(r)_{\text {Total }}^{\Phi^{* 0} / \hat{\Phi}^{* 0}}=\frac{1}{2} g_{\mathcal{D D V}} g_{\mathcal{D}^{*} \mathcal{D}^{*} \mathbb{V}}\left[\frac{X\left[\Lambda, m_{\rho}, r\right]}{4 m_{D} m_{D^{*}} m_{\rho}^{2}}+Y\left[\Lambda, m_{\rho}, r\right]\right]-\frac{1}{2} g_{\mathcal{D D V}} g_{\mathcal{D}^{*} \mathcal{D}^{*} \mathbb{V}}\left[\frac{X\left[\Lambda, m_{\omega}, r\right]}{4 m_{D} m_{D^{*}} m_{\omega}^{2}}+Y\left[\Lambda, m_{\omega}, r\right]\right] \\
& -c\left\{-\frac{g_{\mathcal{D}^{*} \mathcal{D P}}^{2}}{24 m_{D} m_{D^{*}}} U(r)-\frac{g_{\mathcal{D}^{*} \mathcal{D P}}^{2}}{72 m_{D^{2}} m_{D^{*}}} Z\left[\Lambda, \mu^{\prime}, r\right]-\frac{4}{3} f_{\mathcal{D}^{*} \mathcal{D V}}^{2} Z\left[\Lambda, \zeta_{\rho}, r\right]+\frac{4}{3} f_{\mathcal{D}^{*} \mathcal{D V}}^{2} Z\left[\Lambda, \zeta_{\omega}, r\right]\right\} \\
& -g_{\sigma}^{2} Y\left[\Lambda, m_{\sigma}, r\right] \text {, } \\
& \mathcal{V}(r)_{\text {Total }}^{\Phi_{s 1}^{* 0} / \hat{\Phi}_{s 1}^{* 0}}=-g_{\mathcal{D D V} \mathbb{D ^ { * }} \mathcal{D}^{*} \mathbb{V}}\left[\frac{X\left[\Lambda, m_{\phi}, r\right]}{4 m_{D_{s}} m_{D_{s}^{*}} m_{\phi}^{2}}+Y\left[\Lambda, m_{\phi}, r\right]\right]-c \frac{8}{3} f_{D^{*} D \mathcal{V}}^{2} Z\left[\Lambda, \zeta_{\phi}, r\right]+c \frac{g_{\mathcal{D}^{*} \mathcal{D P}}^{2}}{18 m_{D_{s}} m_{D_{s}^{*}}} Z\left[\Lambda, \mu^{\prime}, r\right] \\
& \mathcal{V}(r)_{\text {Total }}^{\Phi_{8}^{* 0} / \hat{\Phi}_{8}^{* 0}}=-\frac{3}{2} g_{\mathcal{D D V} \mathbb{V}} g_{\mathcal{D} * \mathcal{D} * \mathbb{V}}\left[\frac{X\left[\Lambda, m_{\rho}, r\right]}{4 m_{D} m_{D^{*}} m_{\rho}^{2}}+Y\left[\Lambda, m_{\rho}, r\right]\right]-\frac{1}{2} g_{\mathcal{D D V} \mathbb{V}} g_{\mathcal{D} * \mathcal{D} * \mathbb{V}}\left[\frac{X\left[\Lambda, m_{\omega}, r\right]}{4 m_{D} m_{D^{*}} m_{\omega}^{2}}+Y\left[\Lambda, m_{\omega}, r\right]\right] \\
& -c\left\{\frac{g_{\mathcal{D}^{*} \mathcal{D P}}^{2}}{8 m_{D} m_{D^{*}}} U(r)-\frac{g_{\mathcal{D}^{*} \mathcal{D P}}^{2}}{72 m_{D^{\prime}} m_{D^{*}}} Z\left[\Lambda, \mu^{\prime}, r\right]+4 f_{\mathcal{D}^{*} \mathcal{D} \mathbb{V}}^{2} Z\left[\Lambda, \zeta_{\rho}, r\right]+\frac{4}{3} f_{\mathcal{D}^{*} \mathcal{D} \mathbb{V}}^{2} Z\left[\Lambda, \zeta_{\omega}, r\right]\right\} \\
& -g_{\sigma}^{2} Y\left[\Lambda, m_{\sigma}, r\right]
\end{aligned}
$$

The parameter $c$ is $\mp 1$ for the $\mathrm{P}-\mathrm{V}$ systems with the positive and negative charge parity respectively. As expected, the potential of $\Phi^{* 0}$ and $\hat{\Phi}^{* \pm}$ are the same as that of $\Phi^{* \pm}$ and $\hat{\Phi}^{* 0}$ respectively.

\section{B. Numerical results for the P-V system}

We show the variation of the potential with $r$ in Fig. 10. The total effective potential for $\hat{\Phi}^{* \pm}\left(\hat{\Phi}^{* 0}\right)$ and $\Phi_{8}^{* 0}$ is attractive while the potential of $\Phi^{* \pm}\left(\Phi^{* 0}\right)$ and $\hat{\Phi}_{8}^{* 0}$ is repulsive with $\Lambda=1 \mathrm{GeV}$. Especially the neutral states with positive charge parity are very interesting. The only candidate of $X(3872)$ with the attractive potential is $\Phi_{8}^{* 0}$. We collect the numerical results in Table XI. There do not exist molecular states $\Phi^{ \pm}\left(\Phi^{* 0}\right), \Omega^{ \pm}\left(\Omega^{* 0}\right)$ and $\hat{\Omega}_{8}^{* 0}$ because of the strong repulsive potential. Bound state solutions exist for $\Phi_{s}^{* \pm}\left(\Phi_{s}^{* 0}, \bar{\Phi}_{s}^{* 0}\right)$ and $\Omega_{s}^{* \pm}\left(\Omega_{s}^{* 0}, \bar{\Omega}_{s}^{* 0}\right), \hat{\Omega}^{* \pm}\left(\hat{\Omega}^{* 0}\right)$ only with a very large $\Lambda$. We show the dependence of the binding energy of $\Phi_{s 1}^{0}$ on $\Lambda$ in Fig. [11, Moreover we collect the numerical results in Table XII when all coupling constants are increased by a factor of two. When all the coupling constants are reduced by a factor of two, the results are shown in Table XIII.

\section{DISCUSSION AND CONCLUSION}

We have systematically studied the P-P, V-V and P-V systems, which is composed of a pair of heavy meson and anti-meson. We summarize our numerical results from Secs. IV]VI in Tables XIV] XV] and XVI] We use the symbols ' $O$ ' and ' $\otimes$ ' to indicate whether a molecular state exists or not with the coupling constants in Section III] In the following two cases, we tend to label the system with the symbol ' $\otimes$ ': (1) there does not exist a bound state at all because of the repulsive potential; (2) a bound state solution exists only with either $\Lambda>3 \mathrm{GeV}$ or $\Lambda<0.5 \mathrm{GeV}$. If a bound state exists when $0.5<\Lambda<0.9 \mathrm{GeV}$ or $2.0<\Lambda<3.0 \mathrm{GeV}$, we mark this state with '?'. In this case the result is so sensitive to the cutoff parameter that we can not draw a definite conclusion. Only when a loosely molecular state exists with $0.9<\Lambda<2.0 \mathrm{GeV}$, we tend to label the state with the symbol ' $\bigcirc$ '. We want to emphasize the above criteria may be biased due to the authors' personal judgement. The readers are encouraged to consult the numerical results in Sections [V][V] and draw their own conclusions. 


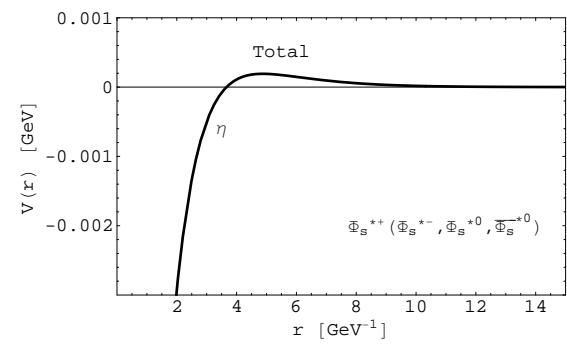

(a)

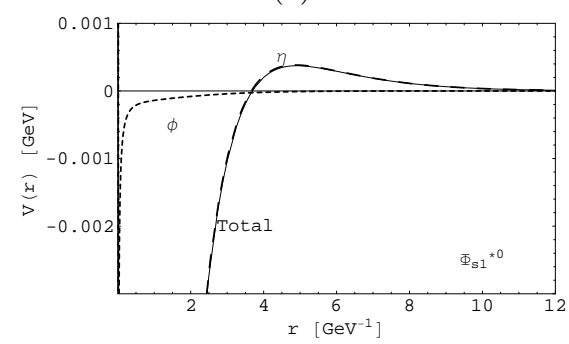

(d)

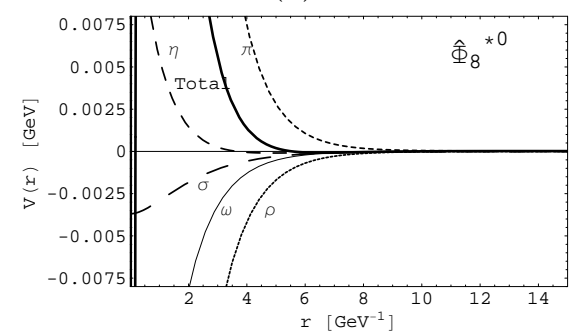

(g)

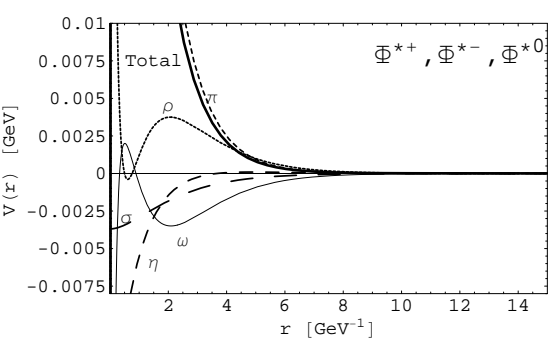

(b)

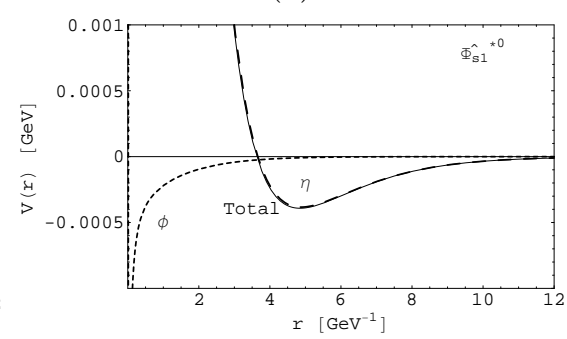

(e)

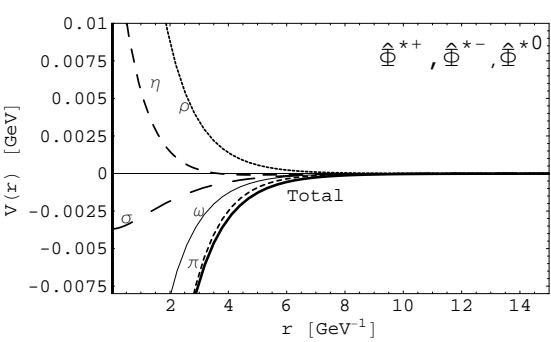

(c)

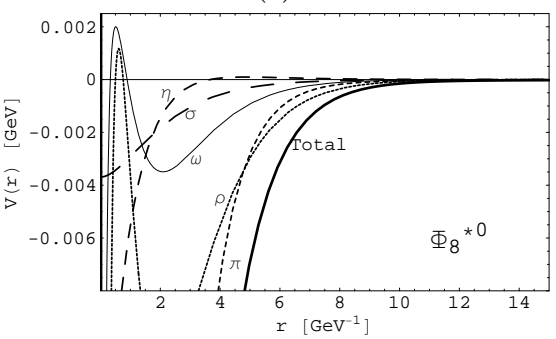

(f)

FIG. 10: The exchange potential of the P-V system with the typical value $\Lambda=1 \mathrm{GeV}$. The thick solid line is the total effective potential.

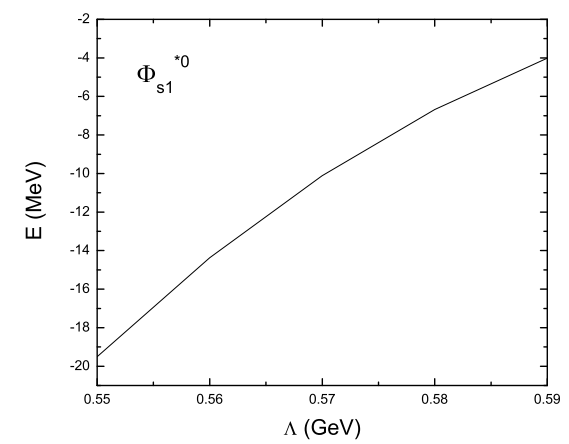

FIG. 11: The dependence of $E$ on $\Lambda$ for $\Phi_{s 1}^{* 0}$.

It's interesting to compare our results with those near-threshold structures observed recently.
1. $Z^{+}(4051)$ 


\begin{tabular}{|c|c|c|c|}
\hline & \multicolumn{3}{|c|}{$\mathcal{D}-\overline{\mathcal{D}}^{*}$} \\
\hline State & $\Lambda(\mathrm{GeV})$ & $E(\mathrm{MeV})$ & $r_{\mathrm{rms}}(\mathrm{fm})$ \\
\hline$\hat{\Phi}_{s}^{* \pm}, \hat{\Phi}_{s}^{* 0}, \hat{,} \Phi_{s}^{* 0}$ & - & - & - \\
\hline$\Phi_{s}^{* \pm}, \Phi_{s}^{* 0}, \Phi_{s}^{* 0}$ & $\begin{array}{l}8.90 \\
9.00\end{array}$ & $\begin{array}{l}-11.74 \\
-25.38\end{array}$ & $\begin{array}{l}1.29 \\
0.88\end{array}$ \\
\hline$\hat{\Phi}^{* \pm}, \hat{\Phi}^{* 0}$ & 10.5 & -12.86 & 1.35 \\
\hline$\Phi^{* \pm}, \Phi^{* 0}$ & - & - & - \\
\hline$\Phi_{s 1}^{* 0}$ & $\begin{array}{l}0.55 \\
0.57\end{array}$ & $\begin{array}{l}-19.51 \\
-10.11\end{array}$ & $\begin{array}{l}1.55 \\
1.93\end{array}$ \\
\hline$\hat{\Phi}_{s 1}^{* 0}$ & $\begin{array}{l}0.60 \\
0.62\end{array}$ & $\begin{array}{c}-17.96 \\
-6.94\end{array}$ & $\begin{array}{l}1.43 \\
2.01\end{array}$ \\
\hline$\Phi_{8}^{* 0}$ & $\begin{array}{l}0.52 \\
0.54 \\
\end{array}$ & $\begin{array}{l}-13.02 \\
-5.25 \\
\end{array}$ & $\begin{array}{l}1.79 \\
2.38 \\
\end{array}$ \\
\hline$\hat{\Phi}_{8}^{* 0}$ & $\begin{array}{l}0.50 \\
0.52\end{array}$ & $\begin{array}{c}-28.61 \\
-9.42\end{array}$ & $\begin{array}{l}1.30 \\
1.91\end{array}$ \\
\hline & & $\mathcal{B}-\mathcal{B}^{*}$ & \\
\hline State & $\Lambda(\mathrm{GeV})$ & $E(\mathrm{MeV})$ & $r_{\mathrm{rms}}(\mathrm{fm})$ \\
\hline$\hat{\Omega}_{s}^{* \pm}, \hat{\Omega}_{s}^{* 0}, \hat{\bar{\Omega}}_{s}^{* 0}$ & - & - & - \\
\hline$\overline{\Omega_{s}^{* \pm}}, \Omega_{s}^{* 0}, \Omega_{s}^{* 0}$ & $\begin{array}{l}3.80 \\
3.90 \\
\end{array}$ & $\begin{array}{l}-3.07 \\
-20.23 \\
\end{array}$ & $\begin{array}{l}2.20 \\
1.00 \\
\end{array}$ \\
\hline$\widehat{\Omega}^{* \pm}, \hat{\Omega}^{* 0}$ & $\begin{array}{l}2.80 \\
3.00 \\
\end{array}$ & $\begin{array}{c}-4.20 \\
-21.13 \\
\end{array}$ & $\begin{array}{l}1.41 \\
0.96 \\
\end{array}$ \\
\hline$\Omega^{* \pm}, \Omega^{* 0}$ & - & - & - \\
\hline$\Omega_{s 1}^{* 0}$ & $\begin{array}{l}2.10 \\
2.20\end{array}$ & $\begin{array}{l}-5.94 \\
-9.79 \\
\end{array}$ & $\begin{array}{l}1.99 \\
1.65\end{array}$ \\
\hline$\hat{\Omega}_{s 1}^{* 0}$ & $\begin{array}{l}0.72 \\
0.73\end{array}$ & $\begin{array}{l}-12.40 \\
-5.95\end{array}$ & $\begin{array}{l}1.59 \\
2.08\end{array}$ \\
\hline$\Omega_{8}^{* 0}$ & $\begin{array}{l}0.92 \\
0.94 \\
\end{array}$ & $\begin{array}{l}-10.19 \\
-18.67 \\
\end{array}$ & $\begin{array}{l}1.13 \\
1.03 \\
\end{array}$ \\
\hline$\hat{\Omega}_{8}^{* 0}$ & - & - & - \\
\hline
\end{tabular}

TABLE XI: The possible bound state solutions for the P-V system.

The charged broad structure $Z^{+}(4051)$ was observed by Belle in the $\pi^{+} \chi_{c 1}$. Its central mass value is slightly above the $D^{*} \bar{D}^{*}$ threshold. First we want to emphasize that there exists strong attraction in the range $r<1$ fm for the $D^{*} \bar{D}^{*}$ system with $J=0,1$ as can be seen clearly from the shape of the total effective potential in Fig. [5] (a)-(b).

If this enhancement is a molecular state, the potential candidate of $Z^{+}(4051)$ is the $\Phi^{* *+}$ state with isospin $I=1$. Our numerical results indicate that there exists a bound state solution (1) for $\Phi^{* *+}$ with $J^{P}=0^{+}$and $\Lambda \sim 4 \mathrm{GeV}$; $(2)$ for $\Phi^{* *+}$ with $J^{P}=1^{+}$and $\Lambda \sim 10 \mathrm{GeV}$. Unfortunately such a cutoff seems too large according to our criteria.

If future experiments confirm $Z^{+}(4051)$ as a loosely bound molecular state, its quantum number is very probably $J^{P}=0^{+}$, which can be measured experimentally through the angular momentum distribution of $\pi^{+} \chi_{c 1}$. Since $\Phi^{* *+}$ belongs to an isospin triplet, its neutral partner state $\Phi^{* * 0}$ may be searched in the $\pi^{0} \chi_{c 1}$ channel.

2. $X(3872)$

In our previous work [15], we explored the $D^{0} \bar{D}^{* 0}$ molecular state by considering the $\pi$ and $\sigma$ meson exchange potentials only. Our numerical results showed that it is impossible to form a $D^{0} \bar{D}^{* 0}$ molecular state with the coupling constants determined by experiment and a reasonable cutoff around 1 GeV [15]. Unfortunately we missed a minus sign in the sigma meson exchange potential, although its contribution is not large. Moreover we didn't take into account the charged modes.

In this work, we consider both the neutral and charged modes and include the exchange force from the $\pi, \eta$, $\sigma, \rho$ and $\omega$ mesons. The resulting total effective potential is attractive as shown in Fig. 10 (f). The vector meson exchange especially the rho meson exchange provides the additional strong attraction. The $\Phi_{8}^{* 0}$ state corresponds the $X(3872)$. 


\begin{tabular}{|c|c|c|c|}
\hline & \multicolumn{3}{|c|}{$\mathcal{D}-\overline{\mathcal{D}}^{*}$} \\
\hline State & $\Lambda(\mathrm{GeV})$ & $E(\mathrm{MeV})$ & $r_{\mathrm{rms}}(\mathrm{fm})$ \\
\hline$\hat{\Phi}_{s}^{* \pm}, \hat{\Phi}_{s}^{* 0}, \hat{\bar{\Phi}}_{s}^{* 0}$ & - & - & - \\
\hline$\Phi_{s}^{* \pm}, \Phi_{s}^{* 0}, \Phi_{s}^{* 0}$ & $\begin{array}{l}2.80 \\
2.90\end{array}$ & $\begin{array}{c}-1.58 \\
-15.38\end{array}$ & $\begin{array}{l}2.57 \\
1.15\end{array}$ \\
\hline$\hat{\Phi}^{* \pm}, \hat{\Phi}^{* 0}$ & $\begin{array}{l}1.90 \\
2.00\end{array}$ & $\begin{array}{l}-16.33 \\
-26.25\end{array}$ & $\begin{array}{l}1.27 \\
1.03\end{array}$ \\
\hline$\Phi^{* \pm}, \Phi^{* 0}$ & - & - & - \\
\hline$\Phi_{s 1}^{* 0}$ & $\begin{array}{l}0.72 \\
0.73\end{array}$ & $\begin{array}{c}-13.66 \\
-8.65\end{array}$ & $\begin{array}{l}1.65 \\
1.93\end{array}$ \\
\hline$\hat{\Phi}_{s 1}^{* 0}$ & $\begin{array}{l}0.75 \\
0.77\end{array}$ & $\begin{array}{l}-22.85 \\
-4.66\end{array}$ & $\begin{array}{l}1.24 \\
2.24\end{array}$ \\
\hline$\Phi_{8}^{* 0}$ & $\begin{array}{l}0.75 \\
0.78 \\
\end{array}$ & $\begin{array}{l}-28.48 \\
-35.20\end{array}$ & $\begin{array}{l}1.15 \\
1.05\end{array}$ \\
\hline$\hat{\Phi}_{8}^{* 0}$ & $\begin{array}{l}1.21 \\
1.23\end{array}$ & $\begin{array}{c}-4.47 \\
-19.31\end{array}$ & $\begin{array}{l}2.29 \\
1.26\end{array}$ \\
\hline & & $\overline{\mathcal{B}-\overline{\mathcal{B}}^{*}}$ & \\
\hline State & $\Lambda(\mathrm{GeV})$ & $E(\mathrm{MeV})$ & $r_{\mathrm{rms}}(\mathrm{fm})$ \\
\hline$\hat{\Omega}_{s}^{* \pm}, \hat{\Omega}_{s}^{* 0}, \hat{\bar{\Omega}}_{s}^{* 0}$ & - & - & - \\
\hline$\overline{\Omega_{s}^{* \pm}, \Omega_{s}^{* 0}, \overline{\Omega_{s}^{* 0}}}$ & $\begin{array}{l}1.60 \\
1.70 \\
\end{array}$ & $\begin{array}{c}-6.24 \\
-44.08\end{array}$ & $\begin{array}{l}1.74 \\
0.74 \\
\end{array}$ \\
\hline$\hat{\Omega}^{* \pm}, \hat{\Omega}^{* 0}$ & $\begin{array}{l}0.87 \\
0.90\end{array}$ & $\begin{array}{c}-5.65 \\
-12.67\end{array}$ & $\begin{array}{l}1.12 \\
1.03\end{array}$ \\
\hline$\Omega^{* \pm}, \Omega^{* 0}$ & - & - & - \\
\hline$\Omega_{s 1}^{* 0}$ & $\begin{array}{l}1.15 \\
1.20\end{array}$ & $\begin{array}{c}-5.70 \\
-30.52\end{array}$ & $\begin{array}{l}1.88 \\
0.96\end{array}$ \\
\hline$\hat{\Omega}_{s 1}^{* 0}$ & $\begin{array}{l}0.82 \\
0.84\end{array}$ & $\begin{array}{c}-34.13 \\
-3.57\end{array}$ & $\begin{array}{l}1.07 \\
2.43\end{array}$ \\
\hline$\widehat{\Omega}_{8}^{* 0}$ & - & - & - \\
\hline
\end{tabular}

TABLE XII: The possible bound state solutions for the P-V system after increasing all the coupling constants by a factor of two.

Recall that the sigma meson exchange force is weak and the pion meson exchange alone does not bind the $D^{*} \bar{D}$ into a molecular state with a physical pionic coupling constant and a reasonable cutoff [15]. In fact a bound state appears only with $\Lambda \sim 6 \mathrm{GeV}$ there. With the additional strong attraction from the vector meson exchange, our present numerical analysis shows that a molecular state exists with $\Lambda \sim 0.55 \mathrm{GeV}$. If we arbitrarily increase all the coupling constants by a factor of two, there exists a molecular state with a slightly more reasonable $\Lambda \sim 0.75$ $\mathrm{GeV}$. If we reduce all the coupling constants by a factor two, there appears a bound state with $\Lambda \sim 0.45 \mathrm{GeV}$. Such a cutoff value is below $1 \mathrm{GeV}$ and smaller than the exchanged vector meson mass.

The pionic coupling constant of the $D^{*} \bar{D}$ system was extracted from the decay width of the $D^{*}$ meson experimentally. Its value is known quite reliably. In contrast, the vector coupling constant was estimated using the vector dominance model in Ref. [28], which may be overestimated according to a recent light cone QCD sum rule analysis [30]. If we fix the pionic and scalar coupling constants but reduce the vector coupling by a factor of two only, there exists a very reasonable bound state solution with $E=-1.75 \mathrm{GeV}$ and $\Lambda=1.4 \mathrm{GeV}$ as shown in Table XVII. Now the value of its root-mean-square radius reaches $3.53 \mathrm{fm}$ ! In other words, such a molecular state is bound very loosely.

In short summary, $X(3872)$ may be accommodated as a molecular state dynamically, although drawing a very definite conclusion is very difficult especially when other available experimental information is taken into account. In our calculation, we assumed the $\mathrm{SU}(2)$ symmetry and ignored the mass difference between the charged and neutral mesons. Moreover we ignored the possible S-D mixing effect completely since we impose the S-wave condition in the derivation of the potential. These approximations together with the sensitivity of the numerical results to the cutoff parameter should be considered in the future investigation.

3. $X(3764)$ 


\begin{tabular}{|c|c|c|c|}
\hline & \multicolumn{3}{|c|}{$\mathcal{D}-\overline{\mathcal{D}}^{*}$} \\
\hline State & $\Lambda(\mathrm{GeV})$ & $E(\mathrm{MeV})$ & $r_{\text {rms }}(\mathrm{fm})$ \\
\hline$\hat{\Phi}_{s}^{* \pm}, \hat{\Phi}_{s}^{* 0}, \hat{\bar{\Phi}}_{s}^{* 0}$ & - & - & - \\
\hline$\Phi_{s}^{* \pm}, \Phi_{s}^{* 0}, \Phi_{s}^{* 0}$ & - & - & - \\
\hline$\hat{\Phi}^{* \pm}, \hat{\Phi}^{* 0}$ & - & - & - \\
\hline$\Phi^{* \pm}, \Phi^{* 0}$ & $\begin{array}{l}0.36 \\
0.37\end{array}$ & $\begin{array}{c}-11.10 \\
-7.30\end{array}$ & $\begin{array}{l}2.07 \\
2.38\end{array}$ \\
\hline$\Phi_{s 1}^{* 0}$ & $\begin{array}{l}0.39 \\
0.41\end{array}$ & $\begin{array}{c}-17.22 \\
-9.31\end{array}$ & $\begin{array}{l}1.74 \\
2.11\end{array}$ \\
\hline$\hat{\Phi}_{s 1}^{* 0}$ & $\begin{array}{l}0.43 \\
0.45\end{array}$ & $\begin{array}{c}-12.33 \\
-5.42\end{array}$ & $\begin{array}{l}1.78 \\
2.32\end{array}$ \\
\hline$\Phi_{8}^{* \sigma}$ & $\begin{array}{l}0.44 \\
0.46\end{array}$ & $\begin{array}{c}-18.01 \\
-8.66\end{array}$ & $\begin{array}{l}1.68 \\
2.11 \\
\end{array}$ \\
\hline$\hat{\Phi}_{8}^{* 0}$ & $\begin{array}{l}0.38 \\
0.40\end{array}$ & $\begin{array}{c}-13.56 \\
-6.17\end{array}$ & $\begin{array}{l}1.90 \\
2.41\end{array}$ \\
\hline & \multicolumn{3}{|c|}{$\mathcal{B}-\mathcal{B}^{*}$} \\
\hline State & $\Lambda(\mathrm{GeV})$ & $E(\mathrm{MeV})$ & $r_{\mathrm{rms}}(\mathrm{fm})$ \\
\hline$\hat{\Omega}_{s}^{* \pm}, \hat{\Omega}_{s}^{* 0}, \hat{\bar{\Omega}}_{s}^{* 0}$ & - & - & - \\
\hline $\bar{\Omega}_{s}^{* \pm}, \Omega_{s}^{* 0}, \bar{\Omega}_{s}^{* 0}$ & - & - & \\
\hline$\widehat{\Omega}^{* \pm}, \hat{\Omega}^{* 0}$ & - & - & \\
\hline$\Omega^{* \pm}, \Omega^{* 0}$ & - & - & - \\
\hline$\Omega_{s 1}^{* 0}$ & - & - & \\
\hline$\hat{\Omega}_{s 1}^{* 0}$ & $\begin{array}{l}3.30 \\
3.40 \\
\end{array}$ & $\begin{array}{c}-1.39 \\
-11.03 \\
\end{array}$ & $\begin{array}{l}2.71 \\
1.41 \\
\end{array}$ \\
\hline$\Omega_{8}^{* 0}$ & $\begin{array}{l}0.46 \\
0.48\end{array}$ & $\begin{array}{c}-19.73 \\
-8.10\end{array}$ & $\begin{array}{l}1.50 \\
1.78\end{array}$ \\
\hline$\hat{\Omega}_{8}^{* 0}$ & - & - & - \\
\hline
\end{tabular}

TABLE XIII: The possible bound state solutions for the P-V system after reducing all the coupling constants by a factor of two.

\begin{tabular}{c|c||c|c}
\hline \hline \multicolumn{3}{c}{$\mathcal{D}-\mathcal{D}$} & \multicolumn{2}{c}{$\mathcal{B}-\mathcal{B}$} \\
\hline state & remark & state & remark \\
\hline$\Phi_{s}^{ \pm}, \Phi_{s}^{0}, \Phi_{s}^{0}$ & $\bigotimes$ & $\Omega_{s}^{ \pm}, \Omega_{s}^{0}, \bar{\Omega}_{s}^{0}$ & $\bigotimes$ \\
$\Phi^{ \pm}, \Phi^{0}$ & $\bigotimes$ & $\Omega^{ \pm}, \Omega^{0}$ & $\bigotimes$ \\
$\Phi_{8}^{0}$ & $?$ & $\Omega_{8}^{0}$ & $\bigcirc$ \\
$\Phi_{s 1}^{0}$ & $?$ & $\Omega_{s 1}^{0}$ & $?$ \\
\hline \hline
\end{tabular}

TABLE XIV: Summary of the $\mathcal{D}-\overline{\mathcal{D}}$ and $\mathcal{B}-\overline{\mathcal{B}}$ systems. The symbols $\otimes$ and $\bigcirc$ denote "forbidden" and "allowed" respectively.

The broad structure $X(3764)$ is close to the $D \bar{D}$ threshold and was observed in $e^{+} e^{-} \rightarrow$ hadrons process. Its quantum number is $J^{P}=1^{--}$. In the present case, we need consider the $\mathrm{P}$-wave $\Phi_{8}^{0}$ by adding the centrifugal potential $\ell(1+\ell) /\left(2 \mu r^{2}\right)$ with $\ell=1$ into the potential in Eq. (19). Here $\mu$ is the reduced mass of the system. In Fig. 12, we show the variation of $V(r)_{\text {Total }}^{\phi_{8}^{0}}+3 /\left(2 \mu r^{2}\right)$ with several typical values of $\Lambda$. Our numerical analysis indicates that there does not exist a $\mathrm{P}$-wave $\Phi_{8}^{0}$ from such a potential. In other words, the structure $X(3764)$ is

\begin{tabular}{c|ccc||c|ccc}
\hline \hline \multicolumn{9}{c}{$\mathcal{D}^{*}-\mathcal{D}^{*}$} & \multicolumn{3}{c}{$\mathcal{B}^{*}-\mathcal{B}^{*}$} \\
\hline state & \multicolumn{3}{c}{ remark } & \multicolumn{3}{c}{ remark } \\
\hline & $J^{P}=0^{+}$ & $J^{P}=1^{+}$ & $J^{P}=2^{+}$ & & $J^{P}=0^{+}$ & $J^{P}=1^{+} J^{P}=2^{+}$ \\
\hline$\Phi_{s}^{* * \pm}, \Phi_{s}^{* * 0}, \Phi_{s}^{* * 0}$ & $\bigotimes$ & $\bigotimes$ & $\bigotimes$ & $\Omega_{s}^{* * \pm}, \Omega_{s}^{* * 0}, \Omega_{s}^{* * 0}$ & $?$ & $\bigotimes$ & $\bigotimes$ \\
$\Phi^{* * \pm}, \Phi^{* * 0}$ & $\bigotimes$ & $\bigotimes$ & $\bigotimes$ & $\Omega^{* * \pm}, \Omega^{* * 0}$ & $\bigcirc$ & $?$ & $\bigotimes$ \\
$\Phi_{8}^{* * 0}$ & $?$ & $?$ & $?$ & $\Omega_{8}^{* * 0}$ & $?$ & $?$ & $?$ \\
$\Phi_{s 1}^{* * 0}$ & $?$ & $?$ & $?$ & $\Omega_{s 1}^{* * 0}$ & $?$ & $?$ & $?$ \\
\hline \hline
\end{tabular}

TABLE XV: Summary of the $\mathcal{D}^{*}-\overline{\mathcal{D}}^{*}$ and $\mathcal{B}^{*}-\overline{\mathcal{B}}^{*}$ systems. 


\begin{tabular}{c|c||c|c}
\hline \hline \multicolumn{2}{c}{$\mathcal{D}-\mathcal{D}^{*}$} & \multicolumn{3}{c}{$\mathcal{B}-\mathcal{B}^{*}$} \\
\hline state & remark & state & remark \\
\hline$\Phi_{s}^{* \pm}, \Phi_{s}^{* 0}, \Phi_{s}^{* 0}$ & $\bigotimes$ & $\Omega_{s}^{* \pm}, \Omega_{s}^{* 0}, \Omega_{s}^{* 0}$ & $\bigotimes$ \\
$\hat{\Phi}_{s}^{* \pm}, \hat{\Phi}_{s}^{* 0}, \hat{\bar{\Phi}}_{s}^{* 0}$ & $\bigotimes$ & $\hat{\Omega}_{s}^{* \pm}, \hat{\Omega}_{s}^{* 0}, \overline{\bar{\Omega}}_{s}^{* 0}$ & $\bigotimes$ \\
$\Phi^{* \pm}, \Phi^{* 0}$ & $\bigotimes$ & $\Omega^{* \pm}, \Omega^{* 0}$ & $\bigotimes$ \\
$\hat{\Phi}^{* \pm}, \hat{\Phi}^{* 0}$ & $\bigotimes$ & $\hat{\Omega}^{* \pm}, \hat{\Omega}^{* 0}$ & $\bigotimes$ \\
$\Phi_{s 1}^{* 0}$ & $?$ & $\Omega_{s 1}^{* 0}$ & $?$ \\
$\hat{\Phi}_{s 1}^{* 0}$ & $?$ & $\hat{\Omega}_{s 1}^{* 0}$ & $?$ \\
$\Phi_{8}^{* 0}$ & $?$ & $\Omega_{8}^{* 0}$ & $\bigcirc$ \\
$\hat{\Phi}_{8}^{* 0}$ & $?$ & $\hat{\Omega}_{8}^{* 0}$ & $\bigotimes$ \\
\hline \hline
\end{tabular}

TABLE XVI: Summary of the $\mathcal{D}-\overline{\mathcal{D}}^{*}$ and $\mathcal{B}-\overline{\mathcal{B}}^{*}$ systems.

\begin{tabular}{c|cc}
\hline \hline$\Lambda$ & $E(\mathrm{MeV})$ & $r_{r m s}(\mathrm{fm})$ \\
\hline 1.6 & -12.86 & 1.47 \\
1.5 & -6.07 & 1.98 \\
1.42 & -2.41 & 3.09 \\
1.41 & -2.07 & 3.30 \\
1.40 & -1.75 & 3.53 \\
\hline \hline
\end{tabular}

TABLE XVII: The bound state solutions for the $D^{*}-\bar{D}$ system if we fix the pionic and scalar coupling constants and reduce only the vector coupling constant in Section III by a factor of two.

not a $D \bar{D}$ molecular state.

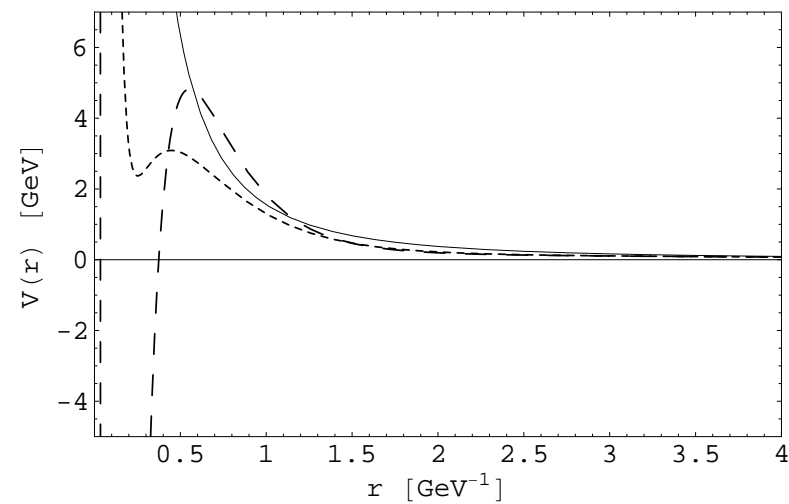

FIG. 12: The dependence of the potential $V(r)_{\text {Total }}^{\phi_{8}^{0}}+3 /\left(2 \mu r^{2}\right)$ on $\Lambda$. The solid, dotted and dashed lines correspond to $\Lambda=1,3,4 \mathrm{GeV}$ respectively.

\section{Acknowledgments}

The authors thank Professors K.T. Chao and Z.Y. Zhang for helpful discussions. This project was supported by National Natural Science Foundation of China under Grants 10625521, 10675008, 10705001, 10775146, 10721063 and China Postdoctoral Science foundation (20070420526). One of authors (X.L.) thanks the support by the Fundação para a Ciência e a Tecnologia of the Ministério da Ciência, Tecnologia e Ensino Superior of Portugal (SFRH/BPD/34819/2007).

[1] Belle Collaboration, K. Abe et al., Phys. Rev. Lett. 100, 142001 (2008).

[2] Belle Collaboration, R. Mizuk et al., arXiv:0806.4098 [hep-ex].

[3] BES Collaboration, M. Ablikim et al., arXiv:0807.0494 [hep-ex]. 
[4] M.B. Voloshin and L.B. Okun, JETP Lett. 23, 333 (1976).

[5] A.D. Rujula, H. Georgi and S.L. Glashow, Phys. Rev. Lett. 38, 317 (1977).

[6] N.A. Törnqvist, Nuovo Cim. A 107, 2471-2476 (1994); Z.Phys. C 61, 525-537 (1994).

[7] M.B. Voloshin, arXiv:hep-ph/0602233.

[8] S. Dubynskiy and M.B. Voloshin, Mod. Phys. Lett. A 21, 2779 (2006).

[9] Y.J. Zhang, H.C. Chiang, P.N. Shen and B.S. Zou, Phys. Rev. D 74, 014013 (2006).

[10] F.E. Close, P.R. Page, Phys. Lett. B 578, 119 (2004).

[11] M.B. Voloshin, Phys. Lett. B 579, 316 (2004).

[12] C.Y. Wong, Phys. Rev. C 69, 055202 (2004).

[13] E.S. Swanson, Phys. Lett. B 588, 189 (2004); ibid B 598, 197 (2004).

[14] N.A. Törnqvist, Phys. Lett. B 590, 209 (2004).

[15] Y.R. Liu, X. Liu, W.Z. Deng and S.L. Zhu, arXiv:0801.3540 [hep-ph], Euro. Phys. J. C 56, 63 (2008).

[16] X. Liu, Y.R. Liu, W.Z. Deng, arXiv:0802.3157.

[17] Y.R. Liu and Z.Y. Zhang, arXiv:0805.1616 [hep-ph].

[18] C.E. Thomas and F.E. Close, arXiv:0805.3653 [hep-ph].

[19] J.L. Rosner, Phys. Rev. D 76, 114002 (2007).

[20] C. Meng and K.T. Chao, arXiv:0708.4222 [hep-ph].

[21] X. Liu, Y.R. Liu, W.Z. Deng and S.L. Zhu, Phys. Rev. D 77, 034003 (2008); arXiv:0711.0494 [hep-ph].

[22] X. Liu, Y.R. Liu, W.Z. Deng and S.L. Zhu, Phys. Rev. D 77, 094015 (2008); arXiv:0803.1295 [hep-ph].

[23] W.M. Yao et al., Particle Data Group, J. Phys. G 33, 1 (2006).

[24] M.P. Locher, Y. Lu, and B. S. Zou, Z. Phys. A 347, 281 (1994) ; X.Q. Li, D.V. Bugg, and B.S. Zou, Phys. Rev. D 55, 1421 (1997); X.G. He, X.Q. Li, X. Liu and X.Q. Zeng, Eur. Phys. J. C 51, 883-889 (2007), arXiv:hep-ph/0606015 X. Liu, X.Q. Zeng and X.Q. Li, Phys. Rev. D 74, 074003 (2006), arXiv: hep-ph/0606191.

[25] H.Y. Cheng, C.Y. Cheung, G.L. Lin, Y.C. Lin, T.M. Yan and H.L. Yu, Phys. Rev. D47, 1030 (1993); T.M. Yan, H.Y. Cheng, C.Y. Cheung, G.L. Lin, Y.C. Lin and H.L. Yu, Phys. Rev. D46, 1148 (1992). M. B. Wise, Phys. Rev. D45, R2188 (1992); G. Burdman and J. Donoghue, Phys. Lett. B 280, 287 (1992).

[26] R. Casalbuoni, A. Deandrea, N. Di Bartolomeo, R. Gatto, F. Feruglio and G. Nardulli, Phys. Rep. 281, 145 (1997).

[27] A.F. Falk and M. Luke, Phys. Lett. B 292, 119 (1992).

[28] C. Isola, M. Ladisa, G. Nardulli and P. Santorelli, Phys. Rev. D 68, 114001 (2003).

[29] V. LEDOUX, M.V. DAELE and G.V. BERGHE, Comp. Phys. Commun. 162, 151 (2004); V. LEDOUX, M.V. DAELE and G.V. BERGHE, ACM Transactions on Mathematical Software 31, 532-554 (2005); http://users.ugent.be/ vledoux/MATSLISE/

[30] Z. H. Li, W. Liu, H. Y. Liu, Phys. Lett. B 659, 598 (2008).

[31] We missed a minus sign in the sigma meson exchange potential in Ref. [15]. 